\title{
The reacting transverse jet in supersonic crossflow: physics and properties
}

\author{
Mirko Gamba, Victor A. Miller, and M. Godfrey Mungal ${ }^{\ddagger}$
}

\begin{abstract}
Combustion in the supersonic regime presents several challenges when compared to its low-speed counterpart. Here we review some of these challenges, and we describe some of the key features of one of the canonical flow fields in supersonic combustion: the reacting transverse jet in a supersonic crossflow (JISCF). From a practical standpoint, the key challenges that limit the control of this combustion regime are fast mixing, robust flame holding and stability. In turn, these aspects are controlled by the complex effects introduced by chemistry, compressibility, shocks and shock/flow interactions, turbulence and the underlying coupling among them. Some of their properties are discussed here. In particular, for a JISCF in a Mach 2.4 high enthalpy crossflow, the reaction zone structure, its dependence on near-wall events, boundary layer, and shock/boundary layer interaction are described. We demonstrate the paramount importance of the coupling between boundary layers and compressibility to provide mechanisms for flame stabilization at the wall. Mixing characteristics, overall structure, and the link to global parameters (momentum flux, velocity and density ratios) that characterize the JISCF, and possibly free shear supersonic flows in general, is also highlighted from non-reacting experiments.
\end{abstract}

\section{Nomenclature}

$d$
$J$
$M$
$p$
$p_{j, o}$
$R e_{d}$
$r$
$s$
$T$
$U$
$x$
$y$
$z$

$=$ Jet exit diameter

$=$ Jet-to-crossflow momentum flux ratio, $\frac{\rho_{\text {jet }} U_{j e t}^{2}}{\rho_{a} U_{a}^{2}}$

$=$ Mach number

$=$ Pressure

$=$ Jet stagnation pressure

$=$ Injector jet exit Reynolds number, $\frac{\rho_{j e t} U_{j e t} d}{\mu_{j e t}}$

$=$ Velocity ratio, $U_{j e t} / U_{a}$

$=$ Density ratio, $\rho_{\text {jet }} / \rho_{a}$

$=$ Temperature

$=$ Speed

$=$ Streamwise direction

$=$ Wall-normal direction

$=$ Spanwise direction

Greek letters:

$\mu \quad=$ Dynamic viscosity

$\rho \quad=$ Density

Subscript:

$a \quad=\quad$ Freestream conditions

jet $=$ Jet conditions

\footnotetext{
*Assistant Professor, Department of Aerospace Engineering, University of Michigan, AIAA Member.

${ }^{\dagger}$ Graduate Student, Mechanical Engineering Department, Stanford University, AIAA Student Member.

‡Professor Emeritus, Mechanical Engineering Department, Stanford University, and Dean of Engineering, Santa Clara University, AIAA Associate Fellow.
} 


\section{Introduction}

$\mathrm{T}$ RANSVERSE jets are formed when a fluid is injected transversely into a stream of air, or simply the crossflow. As a result of the interaction of the jet and crossflow and the entrainment, the jet is deflected and a complex system of vortical structures is formed. ${ }^{1-3}$ For low-speed conditions, the near field is characterized by horseshoe vortices that wrap around the base of the jet and shear layer vortices that develop in the wind-ward side of the transverse jet. The far-field of the jet is dominated by a persistent counter-rotating vortex pair that forms in the near-field and deflects in the far-field to align itself with the direction of the cross-flow. Finally, for sufficiently strong transverse jets, the wake of the jet is characterized by wake vortices that generate near the wall and stretch toward the deflected jet. The supersonic counterpart retains some of these vortical structures, but it also couples with the supersonic crossflow to generate a complex system of shocks and separation regions at the wall. Figure 1 shows schematic diagrams of the flow features of a jet in supersonic crossflow (JISCF). Recent reviews about low-speed transverse jets have been presented by Karagozian ${ }^{4}$ and by Mahesh. ${ }^{5}$

The jet in crossflow (JICF) is one of the most fundamental canonical flows for the investigation of turbulent mixing and combustion. It is relatively simple, yet it retains many features of interest, such as three-dimensionality, separation and recirculation regions, wall-bounded effects, and vortical flow regions. Furthermore, it maintains some level of practical relevance to engineering applications. A jet in crossflow can in fact be found in many technological processes, from industrial level (e.g., an industrial flare stack), to fuel delivery strategies in propulsion applications. A significant body of work has been conducted to characterize and study this system, both at low- and high- (supersonic) speeds, especially under nonreacting conditions. Transverse jets in crossflow has long been a candidate as a simple fuel delivery configuration in supersonic combustion engines. ${ }^{6}$ Arrays of single or staged injectors have in fact been proposed and investigated as fuel delivery strategies for air-breathing high-speed propulsion system ranging form ramjet to scramjet engines. However, the limitations in flame-holding, penetration and mixing characteristics of this configuration have also been identified and different configurations including different mixing-enhancement strategies have been considered. ${ }^{7}$

A typical approach used in the supersonic regime is to deliver a fluid through a sonic underexpanded jet perpendicularly or at a given angle into a supersonic crossflow. A large body of fundamental work has been directed at understanding the general structure of this system, ${ }^{8-14}$ its flowfield and mixing characteristics, ${ }^{15-20}$ the effect of boundary layer ${ }^{8,21}$ and injection details (e.g., angle). ${ }^{22-24}$ Similarly to the underexpanded jets in quiescent environment, ${ }^{9,25}$ transverse underexpanded jets form a complex system of expansion and shock waves (forming the barrel shock) which is deformed by the presence of the crossflow, and it is followed by a jet shear layer that penetrates and spreads into the crossflow. The blockage effect of the issuing jet induces a three-dimensional bow shock wrapping around the transverse jet. For sufficiently large penetration of the jet, the bow shock is nearly normal at the base, folds around the jet and terminates into an oblique shock of diminishing strength. The bow shock stabilized upstream of the jet generates a rise in wall pressure upstream of the jet. ${ }^{15,17,26}$ When this pressure rise is larger than about 3, the boundary layer separates, ${ }^{8}$ thus forming the upstream recirculation region. A three-dimensional oblique separation shock generates as a response to the upstream separation region and it interacts with the bow shock. The properties of the upstream separation region depend on the characteristics of the incoming boundary layer (e.g., laminar or turbulent) and the size of the transverse jet (e.g., diameter) relative to the boundary layer thickness. For instance, the pressure rise in the separation region in a laminar boundary layer is lower than in a turbulent boundary layer, although the length of the separation region is larger by about a factor of three. ${ }^{8}$ Finally, due to the three-dimensionality of the bow shock, the incoming boundary layer experiences an interaction with the bow shock that can extend further away form the immediate vicinity of the injector itself. ${ }^{27,28}$

Previous work considering the JISCF system have investigated its formation, penetration and spreading 9, 11, 12, 29, 30 and its dependance on working conditions, such as the ratio of boundary layer thickness to jet exit diameter. ${ }^{21}$ As for the low-speed (incompressible) counterpart, it has been found that the overall characteristics of the JISCF can be primarily described by the momentum flux ratio $J$ defined as:

$$
J=\frac{\rho_{j e t} U_{j e t}^{2}}{\rho_{a} U_{a}^{2}}=\frac{p_{j e t} \gamma_{j e t} M_{j e t}^{2}}{p_{a} \gamma_{a} M_{a}^{2}}
$$

where $\rho$ and $U$ are the fluid density and speed, respecitvely, $M$ is the Mach number, $p$ is the static pressure and $\gamma$ is the ratio of specific heats. The subscript ' $a$ ' and 'jet' refers to crossflow and jet conditions, respectively. Note that conditions describing the jet properties in equation 1 are taken to be at the jet exit.

Early work on JISCF has considered the distribution of injectant in the wake of the jet ${ }^{18,31,32}$ from which jet penetration has been typically defined. More recent work has investigated the details of the velocity field ${ }^{19}$ and 
furthered the understanding of the mixing field. ${ }^{16,20,33}$ These studies have identified the importance of the wind-ward shear layer on the entrainment process, along with the key role that the counter-rotating vortex pair plays in the wake of the jet in entraining crossflow into center of the jet's wake. Because of the important role that large-scale coherent structures play in the scalar transport process, a large amount of work has been dedicated to understand the dynamics of the these large-scale structures and their interaction with the supersonic crossflow. ${ }^{14,34,35}$ Furthermore, the effect of compressibility (measured by an increase in convective Mach number) on the evolution of large-scale structures and its implications on the decrease in mixing have also been identified. ${ }^{36}$

Reacting transverse jets in the supersonic regime have received less attention, partially due to the stringent requirements to initiate and sustain combustion in a supersonic flow. Several investigations have, however, been directed at developing the capability to investigate the flow field of supersonic reacting flows $\mathrm{s}^{37,38}$ and to investigate the ignition characterstics of hydrogen transverse jets. ${ }^{39-43}$ More recently, work has also been directed at investigate the reacting characteristics of more complex fuel injection approaches that could be found in scramjet applications. ${ }^{44-46}$ Finally, recent numerical efforts have also been dedicated to investigate mixing ${ }^{47,48}$ and ignition of transverse jets. ${ }^{49,50}$

Returning to the scaling of JICF and JISCF, momentum conservation arguments ${ }^{3,51}$ predict that the properties of a JICF are captured by jet-to-crossflow momentum flux ratio scaling laws. Similar models have also been constructed for the supersonic case. ${ }^{29}$ This parameter groups two quantities, the jet-to-crossflow velocity ratio $(r)$ and density ratio $(s)$; thus, $J=s r^{2}$.

As a result, global properties such as the (mean) jet penetration trajectory, velocity and concentration decays are expected to scale with $J$ alone. Experimental observation typically support this reasoning even in the supersonic case. ${ }^{5,52}$ Because these models are based on momentum conservation under the assumption that entrainment and mixing occurs between jet and crossflow fluids, the underlying assumption is that entrainment and small-scale mixing be independent of the detail of the transverse jet, i.e., of $r$ or $s$, but they only depend on the relative momentum, i.e., on $J$. Therefore, under the classical view, two JISCFs with the same values of $J$, but different values of $(s, r)$, should be identical. In particular, they should have the same transverse penetration, which then implies they undergo the same mixing process. This would be the case where two jets are generated by injection of dissimilar fluids at the same $J$; for example, injecting a light or heavy fluid into the supersonic crossflow such that the values of $s$ (and hence $r$ ) are different (i.e., low or large molecular weight, for example hydrogen and argon). However, the limited work available seems to suggest something different, and the current work aims at further exploring this issue.

Recent work ${ }^{53,54}$ in low-speed, low Reynolds number JICF have found that both the velocity and density ratios independently play a significant role on the underlying stability and structure of the JICF that the single parameter $J$ cannot describe. For the supersonic case, previous studies considering injection of dissimilar fluids (at constant $J$ ) have found that the time-average global properties are nearly the same and consistent with a $J$ scaling. ${ }^{14}$ Examples are the penetration and average concentration distribution that are found to be weakly dependent on the injectant. ${ }^{36,55}$ On the other hand, there is also some evidence that the instantaneous structure and coherence of the scalar field may in fact depend on injectant (for example, helium versus air). ${ }^{35,36}$ Furthermore, the characteristics of the velocity field, turbulent fluctuations and intermittent properties also appear to depend on the injectant type. ${ }^{55}$

The current work aims at investigating some of the properties and underlying physics of reacting JISCF. The work combines studies of reacting and non-reacting JISCF where reacting cases are used to identify the flame structure in relation of the system of shock and recirculation regions, while non-reacting cases are used to investigate the dependance of the underlying mixing fields on injection parameters (primary $r$ and $s$ ). In particular, we consider a single, isolated transverse jet injected perpendicularly into a supersonic crossflow characteristic of Mach 8 flight enthalpy for the reacting cases, while we consider a low-enthalpy crossflow for the non-reacting cases.

For the reacting cases, we investigate the effect of $J$ on ignition, stability and combustion characteristics of hydrogen transverse jets. The configuration consists of a flat plate from which hydrogen is injected from an isolated round porthole. The freestream conditions are maintained constant throughout the study at a static pressure and temperature of $40 \mathrm{kPa}$ and $1400 \mathrm{~K}$, respectively, and at a Mach number of 2.4. Different values of $J$ are considered (summarized in table 1) The crossflow is generated by an expansion tube. No active ignition device was used to ignite and stabilize the flame, but ignition of the hydrogen jet was naturally induced by the interaction with the high-enthalpy crossflow. Different diagnostic techniques were used to investigate the system. Primarily, $\mathrm{OH}$ planar laser-induced fluorescence (PLIF) imaging was used to mark the instantaneous reaction zone.

For the non-reacting cases, we explore the interplay of $J, s$, and $r$ on the characteristics, mixing, and structure of JISCFs generated by injecting different fluids at the same value of $J$ but different values of $s$, and $r$. The injection configuration is similar to the one for the reacting cases, but we consider a non-oxidizing low-enthalpy Mach 2.3 crossflow where fluids with different molecular weights were injected at the same value of $J$ (equal to 2.1) to span a range of $(s, r)$ combinations. The investigated parameter space $(s, r)$ at constant $J$ is summarized in table 2 . Toluene 


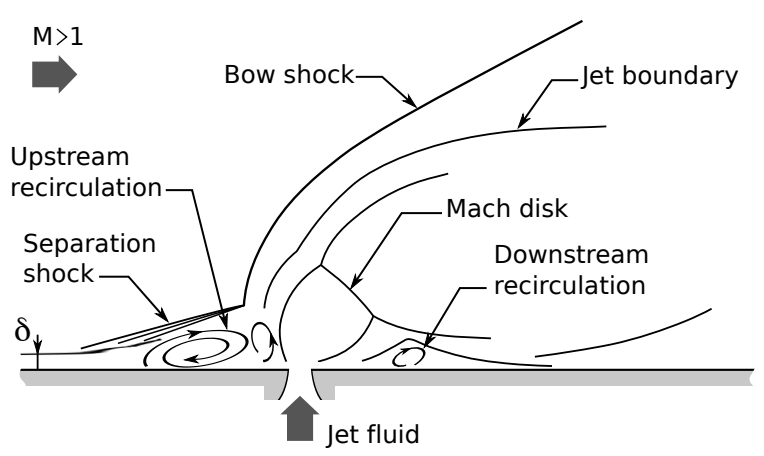

(a)

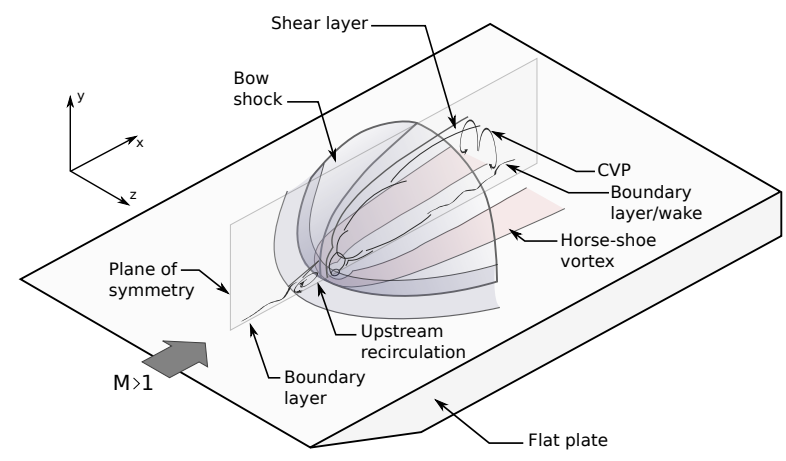

(b)

Figure 1: Schematic diagrams of transverse jet in supersonic crossflow showing some of the dominant flow features. (a) side view; (b) three-dimensional view showing the transverse jet injected from a flat plate.

PLIF temperature imaging ${ }^{56}$ was used to measure the temperature and number density field in the JISCF system as a marker of mixing between (hot) crossflow and (cold) jet fluids. ${ }^{57}$

In the following sections a brief description of the flow facility, experimental configurations and flow diagnostics are given, followed by a description of the main observations on the flowfield, flame structure and mixing properties. The importance of the main flow features that control ignition and flame stabilization processes will be emphasized.

\section{Experimental Setup}

\section{A. Flow facility: the expansion tube}

The experiments presented here were conducted at the 6" Expansion Tube Facility of the High Temperature Gasdynamics Laboratory at Stanford University, which was used to generate the high- and low-enthalpy supersonic crossflows used in all experiments presented here. An account of the design and operation of this facility is given by Heltsley et al. ${ }^{58}$ recent modeling efforts of this facility have been attempted by Örley et al ${ }^{59,60}$ A more detailed accounting of the operation and calibration of the facility to produce the desired conditions used in this studies are also presented elsewhere $27,56,57,61$ and are thus not repeated here in detail. The operational principle and a theoretical analysis of the performance of an expansion tube to generate high-enthalpy supersonic and hypersonic flows can be found in the seminal work of Trimpi. ${ }^{62}$

For the reacting cases, the expansion tube was operated to produce bulk-average freestream conditions of $M_{a}=$ 2.4, $p_{a}=40 \mathrm{kPa}$, and $T_{a} \approx 1400 \mathrm{~K}$ with a test time of about $500 \mu \mathrm{s}$. The freestream was generated using dry air. For the non-reacting cases, the expansion tube was operated to produce a moderate-enthalpy supersonic flow using nitrogen seeded with toluene vapor $(0.5 \%$ by volume) as test gas. To ensure uniform and repeatable seeding, the toluene/nitrogen mixture was prepared manometrically in a separate tank and then used as the test gas in the expansion tube. Throughout this portion of the study, the freestream conditions were maintained constant at $M_{a}=2.3$, $p_{a}=110 \mathrm{kPa}$ and $T_{a} \approx 460 \mathrm{~K}$, with a test time duration of approximately $2 \mathrm{~ms}$. The crossflow conditions reported here were estimated from a set of calibration runs and from a measure of the shock speeds from which nominal, bulk-average conditions were inferred using a zero-dimensional expansion tube solver (based on the expansion tube analysis of Trimpi ${ }^{62}$ ). The data presented here were collected from several single-shot repetitions of the experiments repeated under nominally identical (crossflow) conditions.

\section{B. Transverse jet}

The transverse jet was generated by issuing fluid perpendicularly to the crossflow from a flat plate. A three-dimensional schematic diagram of the flat plate configuration, along with some of the relevant flow features, was shown in figure 1b. The flat plate was approximately $155 \mathrm{~mm}(6.125 \mathrm{in})$ long and $100 \mathrm{~mm}(4 \mathrm{in})$ wide, and it was mounted in the test section within the region of uniform and undisturbed supersonic flow bounded by the limiting characteristic region of the incoming flow and the inviscid core.

For the reacting cases, ultra-high purity grade ( $99.999 \%$ purity) hydrogen was used as fuel. Fuel was injected from a contoured round injector with a $d=2 \mathrm{~mm}$ diameter and located on the centerline of the plate at about $64 \mathrm{~mm}$ 


\begin{tabular}{cccc}
\hline$\left\langle p_{j, o}\right\rangle$ & $s$ & $J$ & $R e_{d}$ \\
{$[k P a]$} & {$[-]$} & {$[-]$} & {$[-]$} \\
\hline 119 & 0.62 & 0.29 & 18,800 \\
289 & 1.50 & 0.71 & 45,800 \\
459 & 2.36 & 1.14 & 72,800 \\
723 & 3.73 & 1.79 & 114,600 \\
1,098 & 5.65 & 2.71 & 174,000 \\
2,034 & 10.7 & 5.03 & 322,400 \\
\hline
\end{tabular}

Table 1: Summary of hydrogen jet injection parameters. $J$ was computed assuming $p_{a}=40 \mathrm{kPa}$. $R e_{d}$ was computed based on cold flow properties of hydrogen at the jet exit plane assuming choked conditions. For all cases, $r=0.69$. Crossflow is held constant at $M_{a}=2.4, p_{a}=40 \mathrm{kPa}$ and $T_{a}=1400 \mathrm{~K}$.

\begin{tabular}{ccccc}
\hline Gas & $s$ & $r$ & $J$ & $R e_{d}$ \\
\hline $\mathrm{H}_{2}$ & 1.46 & 1.2 & 2.1 & $3.3 \times 10^{5}$ \\
$\mathrm{He}$ & 2.72 & 0.89 & 2.1 & $2.1 \times 10^{5}$ \\
$85 \% \mathrm{He} / 15 \% \mathrm{Ar}$ & 6.35 & 0.58 & 2.1 & $3.1 \times 10^{5}$ \\
$\mathrm{~N}_{2}$ & 20.3 & 0.32 & 2.1 & $6.4 \times 10^{5}$ \\
$\mathrm{Air}$ & 20.9 & 0.32 & 2.1 & $6.0 \times 10^{5}$ \\
$\mathrm{Ar}$ & 27.1 & 0.28 & 2.1 & $5.8 \times 10^{5}$ \\
\hline
\end{tabular}

Table 2: Summary of non-reacting jet conditions. Crossflow is held constant at $M_{a}=2.3, p_{a}=110 \mathrm{kPa}$ and $T_{a}=460 \mathrm{~K}$.

from the leading edge. Fuel delivery was controlled by a fast-acting solenoid valve directly mounted to the plenum of the injector. To ensure a fully established flow to the injector, fuel injection was initiated at the beginning of the experiment well before the onset of the flow in the test section. The injector was calibrated by measuring the plenum pressure, $p_{j, o}$, and assuming isentropic expansion to sonic discharge conditions at the exit plane. Six different jets generated at different jet stagnation pressure under the same crossflow conditions were considered in the study. The set of cases is summarize in table 1 and span a range of $J$, from 0.3 to 5.0 at constant freestream conditions. The jet exit Reynolds number, $R e_{d}=\frac{\rho_{j e t} u_{j e t} d}{\mu_{j e t}}$, based on cold properties at the jet exit plane is also reported. For all cases, $r=0.69$ and $s$ varies from 0.6 to 11 .

For the non-reacting cases, the same flat plate system was used. Different values of $s$ and $r$ were investigated by injecting gases with different molecular weights while maintaining a constant value of $J=2.1$. To explore a range of $(s, r)$ pairs, injection of hydrogen, helium, helium/argon (85\%/15\%) mixture, nitrogen, air and argon were considered; these cases correspond to a range $s=1.5-27$, with corresponding values of $r=1.2-0.3$ and $R e_{d} \approx 3 \times 10^{5}-6.5 \times 10^{5}$. The total temperature of the injected fluids was near room temperature $(295 K)$. The cases considered in the study are summarized in table 2 .

\section{Imaging configuration for reacting jets}

In the study different imaging techniques were used to investigate the system: the flow and shock structure was visualized by Schlieren imaging (instantaneous $-500 \mathrm{~ns}$ exposure - and time-averaged - $20 \mathrm{us}$ exposure), the overall characteristics of the reacting jets were investigated by $\mathrm{OH}^{*}$ chemiluminescence imaging, whereas the instantaneous reaction zone was visualized by $\mathrm{OH}$ planar laser-induced fluorescence (PLIF) imaging. For the non-reacting cases, toluene PLIF thermometry was used to measure temperature field as a marker of mixing.

For the reacting cases, OH PLIF imaging of the $Q_{1}\left(N^{\prime \prime}=7\right)$ line near $283.3 \mathrm{~nm}$ of the $A^{2} \Sigma^{+} \leftarrow X^{2} \Pi\left(v^{\prime}=1, v^{\prime \prime}=0\right)$ electronic transition was used to mark the instantaneous reaction region. Broadband fluorescence from the whole upper excited state to all lower states was collected. The excitation source was provided by the frequency-doubled output of a tunable dye laser pumped by a frequency-double Nd:YAG laser operated at $10 \mathrm{~Hz}$. The laser sheet thickness was estimated to be $300 \mu \mathrm{m}$. A gated intensified CCD camera $(512 \times 512$ pixels resolution) fitted with a Nikon $105 \mathrm{~mm}, \mathrm{f} / 4 \mathrm{UV}$ lens was used to collect fluorescence. UG-11 and WG-295 filters were used to reject unwanted light. Several side- and plan-view planes were considered. With reference to the coordinate system defined in figure $1 \mathrm{~b}$, side-view planes are defined to be $x-y$ planes, whereas plan-view planes are defined to be $x-z$ planes. For the $\mathrm{OH}^{*}$ chemiluminescence imaging, an imaging configuration similar to the OH PLIF experiments were used and differed only in the choice of spectral filter. For this case, a bandpass interference filter centered at $313 \mathrm{~nm}$ with a full-width at half-max of $10 \mathrm{~nm}$ was used. Only long exposure $(200 \mu \mathrm{s})$ imaging was considered to obtain a time-averaged and line-of-sight integrated representation of the overall location of the burning regions.

For the non-reacting cases, a measure of mixing was inferred from a measurement of the temperature field because jet and crossflow were at different initial temperatures. Temperature is measured using the single-wavelength exci- 


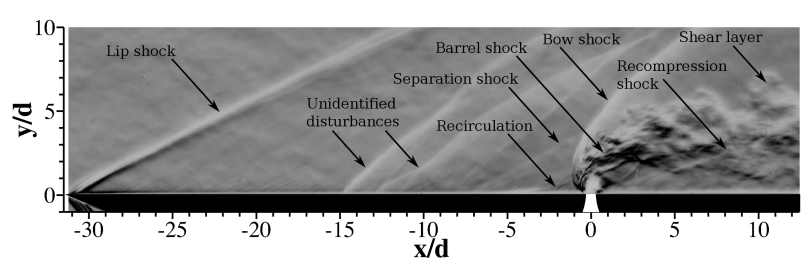

(a)

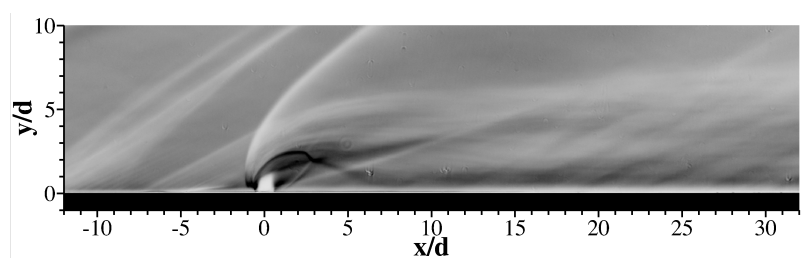

(b)

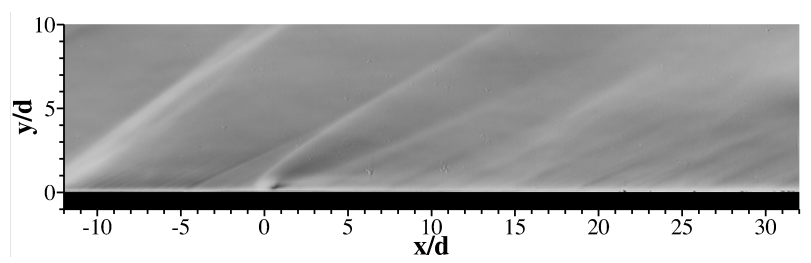

(c)

Figure 2: Schlieren images on the flow and shock structure around transverse underexpanded jets in supersonic crossflow: (a) instantaneous (500 $\mathrm{ns}$ exposure) Schlieren image for $J=5.0$,(b) time-average (20 $\mu \mathrm{s}$ exposure) Schlieren image for $J=5.0$, and (c) $J=0.3$.

tation, dual-band detection toluene PLIF thermometry technique developed and optimization for supersonic flows by Miller et al., ${ }^{56}$ and extended to non-uniform supersonic flows by Gamba et al. ${ }^{57}$ A detailed description of the method can be found in the cited references. For the experiments, the excitation laser light was provided by a frequency quadrupled injection seeded Nd:YAG laser. Fluorescence over two spectral bandwidths was collected with two separate ICCD cameras equipped with suitable collection optics and filters. ${ }^{56}$ The information from each camera was then combined to infer the local temperature and tracer number density ${ }^{57}$ Because the tracer (toluene) was seeded only in the crossflow, the measured number density refers to the local number density of fluid originated from the crossflow.

\section{Results}

\section{A. Reacting cases: flame structure}

\section{Shock structure}

The structure of the shock system for the case of the current experiments is shown in figure 2 where an instantaneous (500 ns exposure) and time-average (20 $\mu s$ exposure) Schlieren images are presented. The main characteristics have been outlined in the schematic diagram of figure 1 and correspond to the features indicated in figure 2. Some of the more relevant shock structures have been labeled in figure 2(a) to aid the interpretation of the images. In all figures presented here, the crossflow is from left to right. Figure 2a shows an instantaneous snap-shot for the $J=5.0$ case. The image captures the incoming flow as it develops over the flat plate (indicated by the black outline) and the near-field of the transverse jet. Figure $2 \mathrm{~b}$ shows the corresponding time-average Schlieren image for the same case of figure 2a. A few jet diameters upstream of the injector, the near-field and part of the wake of the transverse jet are shown. Finally, figure $2 \mathrm{c}$ shows the time-average shock structure generated around a $J=0.3$ case.

The salient JISCF features that the Schlieren images identify are the bow shock curving around the jet, the separation oblique shock, the deformed barrel shock terminating in a tilted Mach disk, the recompression shock at the lee-side of the transverse jet following reattachment, and the shear layer of the jet. Furthermore, these characteristics are strongly influenced by the value of $J$. In particular, the penetration of the barrel shock increases with $J$; similarly, 


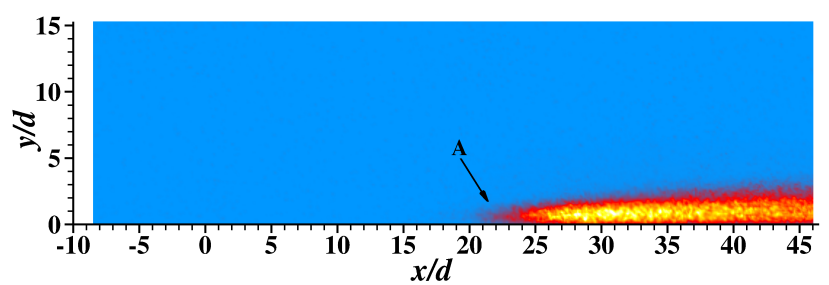

(a)

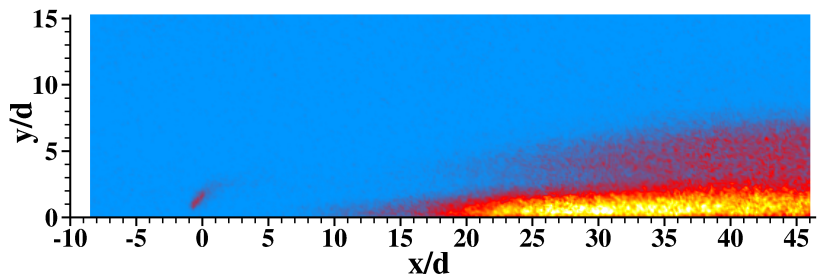

(b)

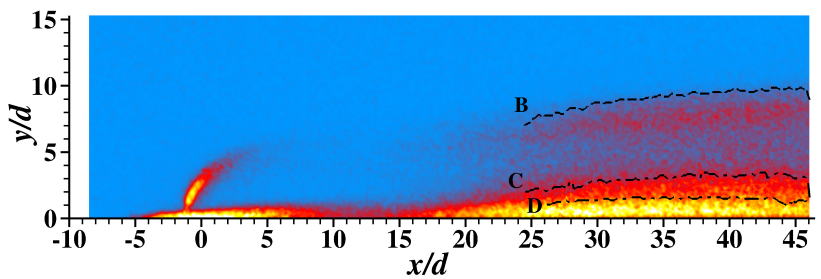

(c)

Figure 3: Line-of-sight integrated, time-averaged $\mathrm{OH}^{*}$ chemiluminescence imaging of the overall flame structure: (a) $J=0.3$, (b) $J=1.8$, and (c) $J=5.0$. For all cases the exposure was set to $200 \mu s$. Labels and lines are described in the text.

the deflection and deformation of the barrel shock system is accentuated as $J$ is lowered until it disappears as it merges with the wake and boundary layer, as for the $J=0.3$ case, or until the subsonic limit is reached. The penetration of the jet into the crossflow provides flow blockage to the incoming flow, hence generates a three-dimensional bow shock whose strength depends on the level of blockage (i.e., the value of $J$ ). The role and importance of the bow shock on the combustion characteristics will be emphasized further below when the global and instantaneous flame structure are discussed. One aspect to note here is the importance of the interaction of the bow shock with the incoming flow, especially with the boundary layer. As a result of the bow shock/boundary layer interaction, the incoming boundary layer separates (a process that is favored by laminar boundary layer ${ }^{8,18,32}$ ) and a recirculation region forms in front of the jet. As a result of the recirculation region, a separation shock forms in front of the separation bubble (see figure 2a). Both features play an important role in flame stabilization and flame-holding. $6,8,9,13,14,29,41$

\section{Global characteristics of the burning transverse jet}

The overall ignition and flame characteristics of the system were investigated using $\mathrm{OH}^{*}$ chemiluminescence imaging from naturally occurring, chemically excited hydroxyl radicals as they decay from the $A^{2} \Sigma^{+}$excited electronic state to the $X^{2} \Pi$ ground state through spontaneous radiative emission. Chemiluminescence imaging is used for a qualitative description of the burning characteristics, their morphology and dependencies. Note that the imaging approach provides a line-of-sight and time integrated representation of the combusting flowfield from a lateral view.

Examples of the time-averaged $\mathrm{OH}^{*}$ chemiluminescence imaged over a portion of the JISCF flowfield and integrated in time over $200 \mu s$ during the second half of the test time are shown in figure 3 for transverse jets at (a) $J=0.3$, (b) $J=1.8$, and (c) $J=5.0$. The colormap used in constructing figure 3 ranges from blue in the nonreacting regions to red and yellow in the reacting ones and terminates with white at the maximum intensity. Note that all conditions are presented on the same scales for consistency.

Inspection of the set of images generated for the selected range of $J$ values reveals that the characteristics of the 
overall burning regions, their structure and the stabilization points depend on the value of $J$. The cases reported in figure 3 are selected to demonstrate the limiting behaviors that the study identifies. In particular, for values of $J<1$ (figure 3a), it is observed that ignition does not occur at the injection point, but the flame is stabilized at some downstream point, which we refer to as the stabilization point $x_{s}$, and it is indicated by the label A in figure $3 \mathrm{a}$. Furthermore, the burning region appears to be confined at the wall in a layer approximately $2 d$ thick. As the Schlieren image of figure 2c qualitatively indicates, this case of low value of $J$ results in minimal fuel penetration into the cross-flow, but the wake of the jet remains attached to the wall.

As the value of $J$ is increased, the jet penetration increases, and the stabilization point moves upstream. Nevertheless, the most intense burning region, as indicated by intense $\mathrm{OH}^{*}$ emission, still remains confined to the near-wall wake region of the transverse jet. As the value of $J$ is raised above unity (see figure $3 \mathrm{~b}$ for the $J=1.8$ case), weak shear layer burning is detected by the $\mathrm{OH}^{*}$ imaging and exists above the intense near-wall wake burning. For the $J=1.8$ case, near-field burning on the wind-ward side of the transverse jet just behind the bow shock is also detected, but it is a weak feature.

Burning of the shear layer, either in the near-field in the region of maximum deflection or downstream in the wake of the jet, becomes important only as $J$ is increased to values larger than $\sim 3$. As the value of $J$ is the increased even further, the downstream stabilization point and the near-field burning merge and the reacting transverse jet is stabilized directly at the injector and upstream of it (see figure 3c). However, even for the large value of $J$ considered in figure $3 \mathrm{c}$, the near-wall wake remains the region with the most intense burning as identified by the $\mathrm{OH}^{*}$ emission. Furthermore, also for the $J=5.0$ case, there exists a region of low $\mathrm{OH}^{*}$ emission downstream of the region of maximum deflection separating the near-field, post-bow-shock burning and the near-wall-stabilized wake combustion. This low $\mathrm{OH}^{*}$ intensity region is interpreted as a quenched region subject to rapid expansion of the transverse jet. Finally, for $J$ larger than $\sim 3$, burning upstream of the injection point is observed (see figure $3 \mathrm{c}$ ). This feature has typically been associated with the upstream recirculation region which ignites and serves as a point of stabilization of the combustion process $^{6}$ and has also been observed in previous work. ${ }^{40,41}$

As a concluding remark, it is important to point out that in all cases considered in the study (variation of $J$ for constant cross-flow conditions) most of the $\mathrm{OH}^{*}$ chemiluminescence emission is detected close to the wall in the wake of the transverse jet, and, on average, emission in the shear layer is weaker, at least within the imaging region. Therefore, under the assumption that $\mathrm{OH}^{*}$ emission tracks the heat release distribution, it appears that the region where most of the heat release occurs might be confined in the wake of the jet near the wall.

The results of the present $\mathrm{OH}^{*}$ imaging experiments suggest that the near field ignition characteristics are strongly coupled to the jet penetration, which is dictated by the value of $J$. The first effect of jet penetration is to provide flow blockage to the incoming flow and the formation of a bow shock at the injection location. The bow shock can couple with the dynamics of the jet itself, but more important, it also interacts with the incoming boundary layer, and the formation of the upstream recirculation region. Because the importance and strength of these effects can be linked to jet penetration, the parameter $J$ might be the governing quantity to describe these effects.

We speculate that for high values of $J$, the strong bow shock/jet interaction dominates the ignition process due to local shock heating and compression, but also due to longer residence time within the stagnation region at the jet; on the contrary, for low values of $J$, the ignition process is driven by mixing of cold jet fluid with hot freestream fluid at a (quasi-)constant pressure ${ }^{8,15,17}$ in a manner that could possibly be a (partially) premixed autoignition process. It is however clear that near-wall events tend to dominate the ignition process, at least for the range of $J$ values considered in the study.

The overall penetration of the transverse jet flame into the crossflow increase as $J$ increases. Penetration of jets in crossflow, both in the subsonic ${ }^{3,63,64}$ and supersonic ${ }^{13,16,29,30,52}$ regimes, is found to be primary described by the parameter $J$, although weak effects on boundary layer thickness, ${ }^{21}$ crossflow Mach number ${ }^{52}$ and jet fluid molecular weight ${ }^{35}$ might be present. If a flame jet penetration is extracted from the $\mathrm{OH}^{*}$ results, the same result approximately holds for these cases as well. ${ }^{27}$ However, our second part of the work on the mixing properties, we will identify that the parameter $J$ might not be sufficient to describe the details of the turbulent structure of the jet and its mixing properties.

\section{Instantaneous reaction zone structure}

OH PLIF imaging helps us investigating the instantaneous flame structure. ${ }^{27}$ Imaging was carried out on several orthogonal planes. In particular, side- and plan-view planes are considered here. Side-view planes are defined as streamwise/wall-normal planes where imaging is carried from the side of the jet; plan-view planes are defined as streamwise/spanwise planes, i.e. planes parallel to the flat plate surface with imaging from above the plate. Different off-centerplane side-view planes and plan-view planes at different heights from the flat plate are considered. Furthermore, in constructed the OH PLIF images shown here, two OH PLIF images (from different runs) corresponding to 


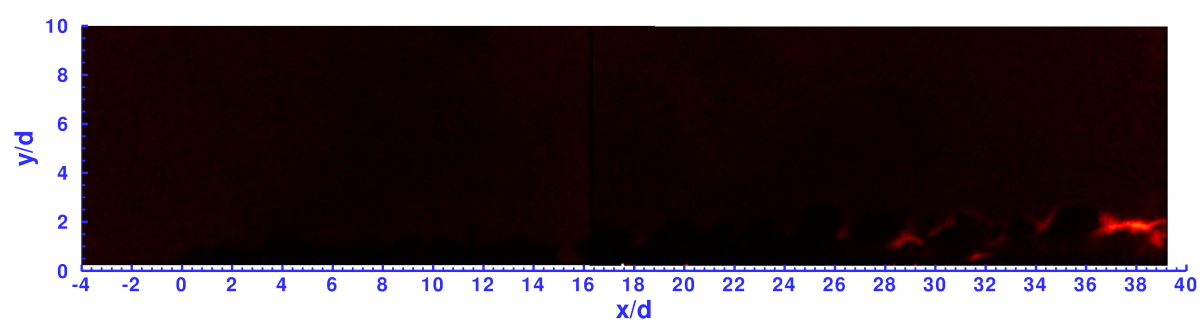

(a)

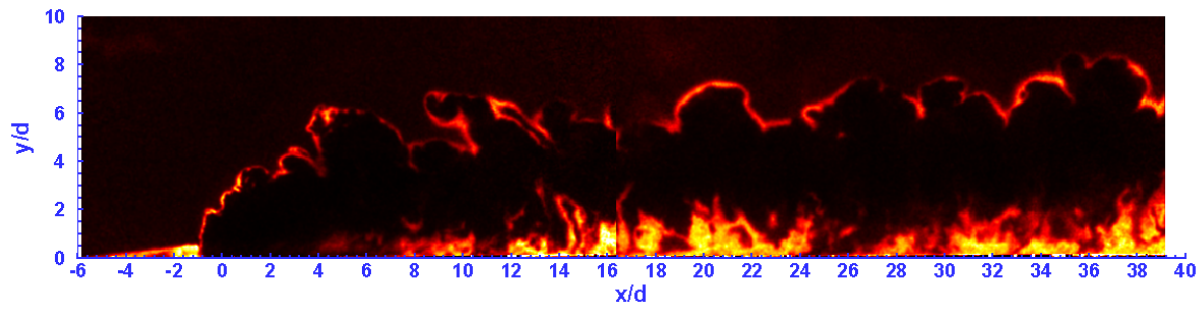

(b)

Figure 4: OH PLIF imaging marking the instantaneous reaction zone on the symmetry plane of the jet (side-view) for the (a) $J=0.3$, and (b) $J=5.0$ JISCF.

two adjacent imaging locations were appropriately combined to cover the full streamwise extent of the flowfield of interest.

Figure 4 shows an example of the instantaneous reaction zone as marked by the OH PLIF for the symmetry centerplane of the transverse jet for: (a) the $J=0.3$ and (b) $J=5.0$ jets. Under the current flow conditions and sufficiently high values of $J$ (figure 4(b)), three major flow features are observed: $(i)$ an intermittently reacting recirculation region upstream of the jet, (ii) a reactive shear layer on the windward side of the transverse jet, and (iii) a highly reactive boundary layer that extends laterally for several diameters off the jet centerplane. Figure 5 shows OH PLIF images on a side-view plane 5 diameters off the centerplane for both the (a) $J=0.3$ and (b) $J=5.0$ jets. The imaging of figure 5 clearly demonstrates the presence of the reactive boundary layer away from the immediate vicinity of the wake of the jet. At low values of $J$ only wake burning near the wall and off the centerplane is a dominant region of burning. On the contrary, shear layer ignition is a weak feature, even on the centerplane of the jet (figure 4(a)). Therefore, it seems reasonable to assume that the intense near-wall burning observed from $\mathrm{OH}^{*}$ chemiluminescence is primarily due to the intense $\mathrm{OH}$ regions near the wall shown in figure 5. Many of the features observed in the side-views of figure 4 are consistent with previous experimental investigations on similar systems. ${ }^{40,65}$ However, contrary to the observations of Ben-Yakar et al., ${ }^{41}$ when ignition occurs upstream or just downstream of the injection point, flame quenching is not observed as the flow expands around the base of the transverse jet.

Plan-view OH PLIF imaging at different wall-normal locations better demonstrate the existence of $\mathrm{OH}$ in the boundary layer near the surface as anticipated by figure 5 . Examples at different wall-normal locations are shown in figures 6 and 7 for the $J=0.3$ and $J=5.0$ case, respectively. Cases corresponding to low $J$ values are characterized by a plan-view imprint of the reaction zone that is consistently similar to the case of figure 6 . They are characterized by: (i) long separation between the injection porthole and the point of ignition which is consistent with the stabilization point location shown by the $\mathrm{OH}^{*}$ images; (ii) a broad reaction zone that is several jet diameters wide, that grows with downstream distance and enlarges with increasing value of $J$; (iii) a characteristic spreading of the $\mathrm{OH}$ zones downstream of the ignition point (the spreading angle consistently increases with $J$ ); and (iv) a narrow region (on the order of 2 jet diameters in size) located along the symmetry plane where burning does not occur at all values of $J$ but the largest one considered. These general characteristics are observed and are similar to some extent for all $J$ values considered, although their relative span-wise size increase with $J$. For example, the broad, diffuse $\mathrm{OH}$ regions at the wall for $J=0.3$ (figure 6) are on the order of $5 d$ wide, and they grow to $10 d$ for $J=1.8$ (not shown). When $J$ is sufficiently large ( $J=5.0$ for this case), it appears that something drastic occurs in the flow-field structure and which results in a significant modification of the near-wall $\mathrm{OH}$ structures (figure 7). As it will be discussed further below, it is believed that the flow structure that leads to the $\mathrm{OH}$ imprint of figure 7(a) is related to the strength of the bow shock and the importance of shock/boundary layer interaction on the near-field flow and mixing characteristics. ${ }^{28}$ 


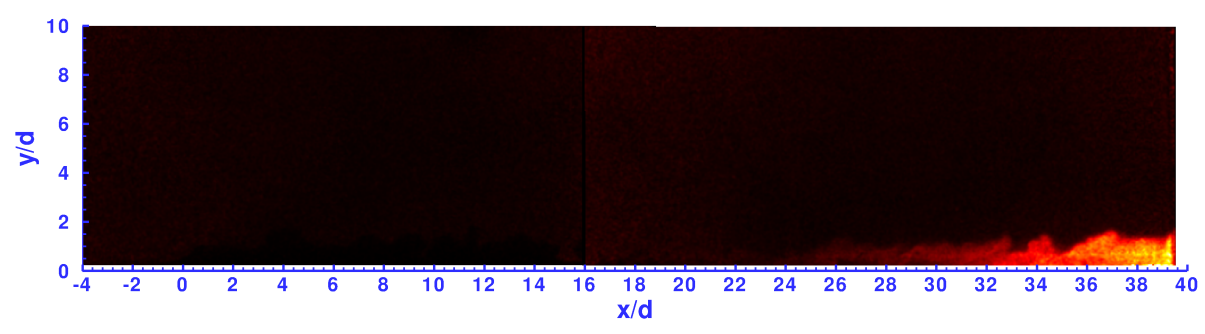

(a)

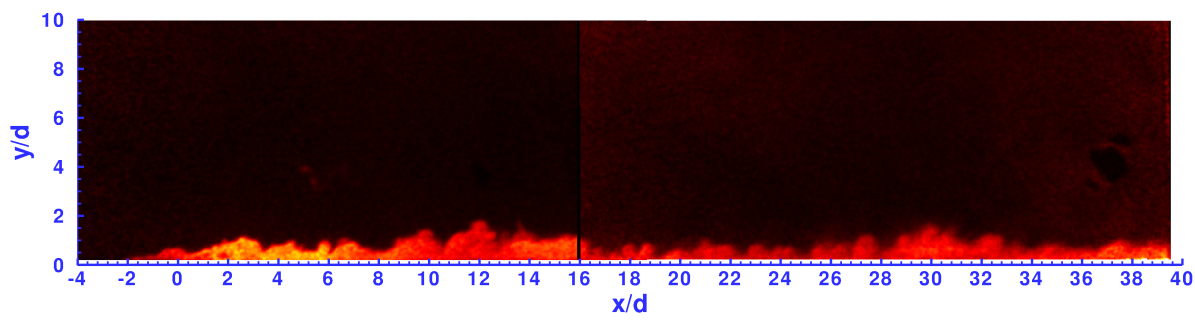

(b)

Figure 5: OH PLIF imaging marking the instantaneous reaction zone on a side-view plane at $z / d=5$ for the (a) $J=0.3$, and (b) $J=5.0 \mathrm{JISCF}$.

Comparing imaging results for the different cases suggests that the thickness of these near-wall regions is relatively independent of the value of $J$, or at most a very weak function of it. In fact, in all cases the edge of these burning regions is located at about $y / d=1$. We speculate that this feature is related to the structure of the incoming boundary layer and its tendency to separate upon interaction with the bow shock. Therefore, we believe that the observed nearwall imprint is an extensive reacting boundary layer where fuel entrainment is sustained by the upstream recirculation region and by the bow shock/jet interaction. ${ }^{28}$

Imaging several diameters off the plate surface detects only the reacting shear layer of the jet (figures 6(d)-7(d)). These reacting regions show characteristics similar to the side-views of figure 4 and they are part of the shear layer system of the main body of the transverse jet. The lateral extent of the jet body is significantly narrower than what observed near the plate, being only a few jet diameters wide. Similarly to low-speed transverse jets, the wake of the jet is dominated by a counter-rotating vortex pair $(\mathrm{CVP})^{19}$ that has a significant role in crossflow entrainment and mixing in the wake of the jet. ${ }^{20}$ For low $J$, the penetration of the jet is only a few jet diameters deep and the evolution of the CVP is inevitably limited by its proximity to the wall. The strength of the interaction of the CVP with the wall and the boundary layer would be expected to increase with diminishing jet penetration $(J)$. The observed near-wall structure of the burning regions, therefore, might also be a result of this interaction.

The set of plan-view $\mathrm{OH}$ imaging indicates that ignition in the recirculation region in front of the transverse jet occurs only at large $J$ (in this case at $J=5.0$ ). However, the mixing study shown below suggest that fuel entrainment in the upstream separation region might exist even at lower values of $J$. As shown in figure 7(a-b), this reacting recirculation region wraps around the base of the bow shock which surrounds the jet, ${ }^{28}$ leaving a region just downstream of the jet where reaction is not observed. The analysis of Huber et al. ${ }^{6}$ on ignition and flameholding capabilities of different wall-injection configurations identified the upstream recirculation as a suitable location for flame stabilization due to the long residence time and near stagnation conditions. Therefore, the length of the upstream recirculation region (which increases with $J$ ), ${ }^{8}$ the low velocities associated with it ${ }^{19}$ and the blockage effect of the jet would all play an important role in this process, especially at large $J$. Another mechanism supporting ignition in the separation region is the favorable mixing that occurs as a result of the interaction of the issuing jet and the system of recirculation regions $6,39,66$ along with the shorter ignition times expected at lean conditions in supersonic nonpremixed combustion of a cold fuel mixed with a hot oxidizer. ${ }^{6}$

Everett et $a l .{ }^{15}$ carried out surface oil-flow visualization around transverse jets $($ at $J=1-2)$ to investigate the streamline pattern resulting from the interaction of the jet with the bow shock and the boundary layer. They identified two separation lines that wrap around the jet and that create two separate regions that do not interact. Between these two separation lines, and between the second separation line and the injector, two recirculation bubbles exist. The 


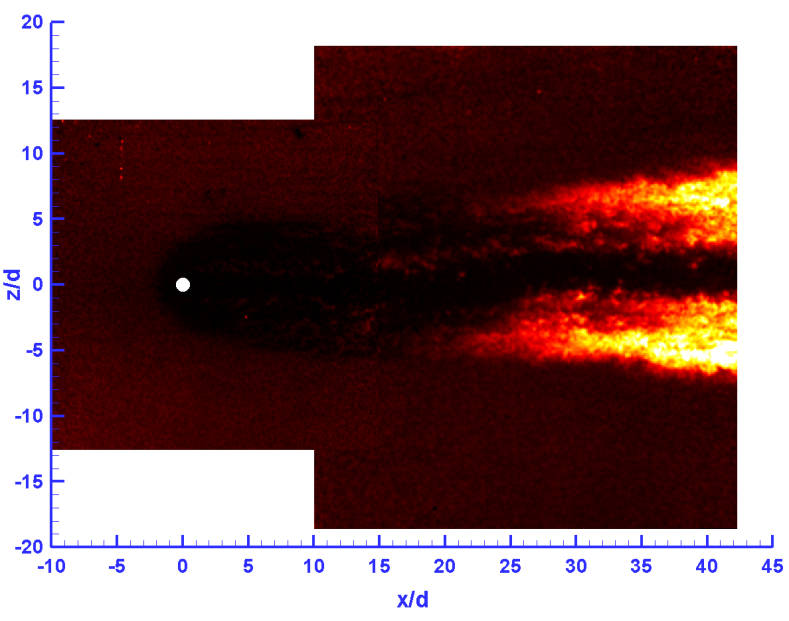

(a)

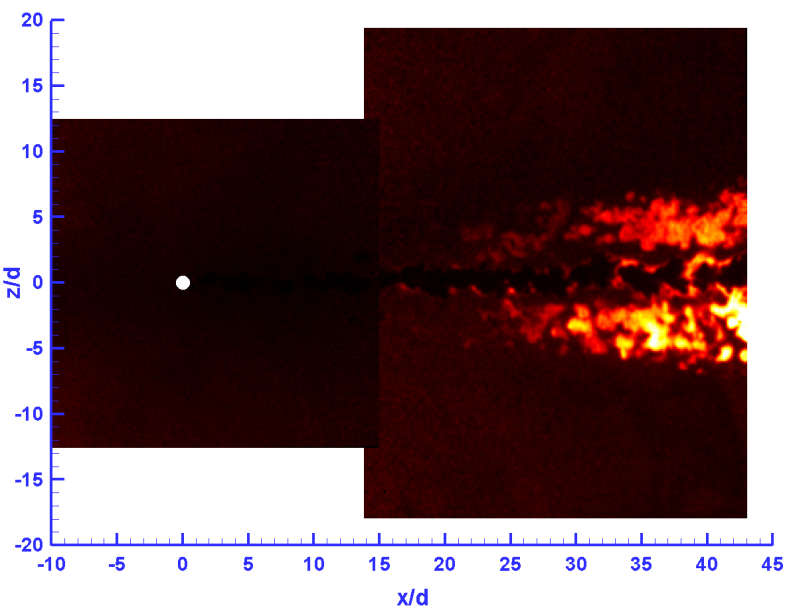

(c)

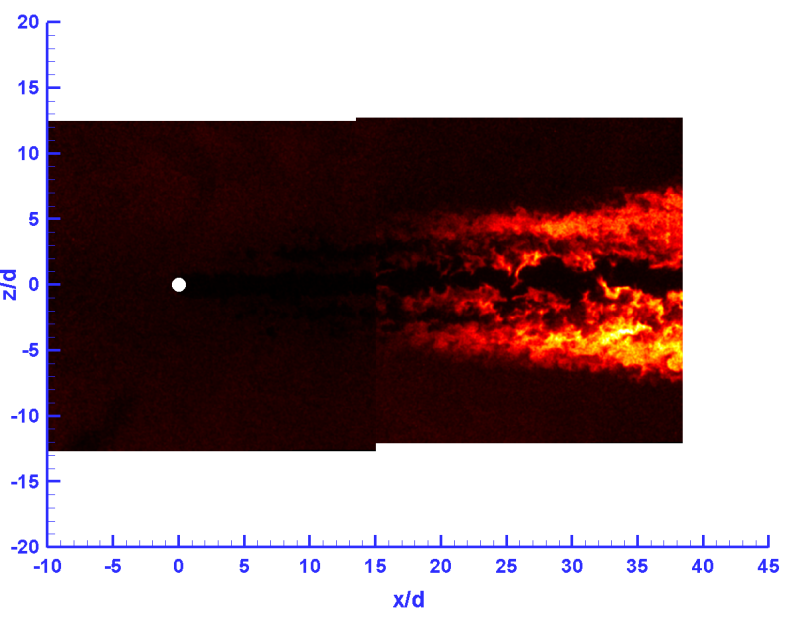

(b)

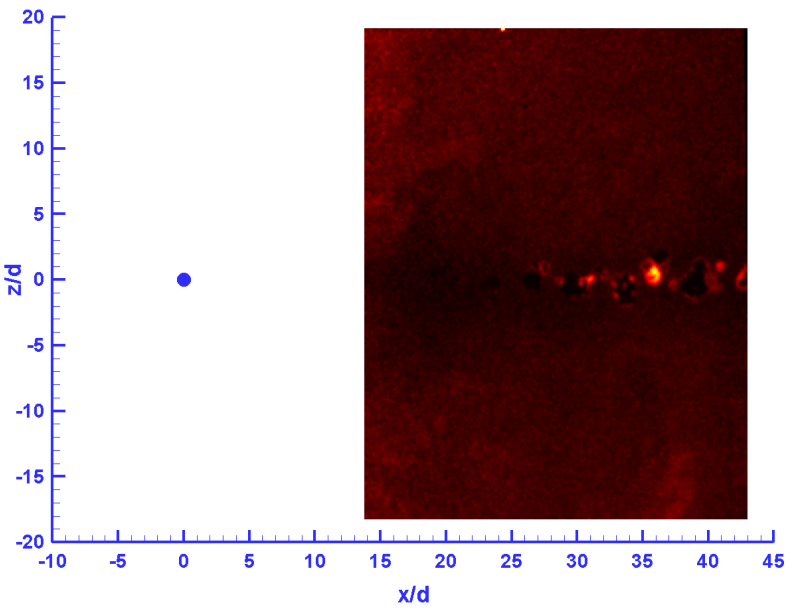

(d)

Figure 6: Instantaneous OH PLIF image on different plan-view planes for the $J=0.3$ JISCF. (a) $y / d=0.25$, (b) $y / d=0.5$, (c) $y / d=1$, and (d) $y / d=2$.

first recirculation region is associated with the boundary layer, whereas the second recirculation region is produced by the issuing jet (recall also figure 1 for a schematic diagram). Everett et al. observed that oil used to mark the wall streamline did not move across these two recirculation systems. However, from the imaging of the current study, it is clear that mass transfer across the two recirculation regions has to occur to provide fuel to sustain combustion within the large interaction region. We will also observe a similar effect on the mixing study further below for a value of $J$ of 2.1, which is closer to the range considered by Everett et al. ${ }^{15}$ in drawing their conclusion. Streamlines at the wall follow the trajectory of the separation streamlines and they all wrap around the base of the jet. The oil-flow visualization of Everett et al. also identified a third recirculation region just downstream of the injector which did not interact with the upstream separation lines. This third recirculation region originates at the injector and extends to a triangular region downstream of the injector that is then swept downstream in the wake of the jet.

Many of the features observed by the oil-flow visualization experiments of Everett $e t$ al. share many similarities with the structure shown in figure 7(a) for the $J=5.0$ case where the instantaneous structure of the $\mathrm{OH}$ is used to mark them (however, the same comparison cannot be conclusively drawn from cases at lower $J$ as the $\mathrm{OH}$ for the most part marks only regions downstream of the injector). For example, the broad $\mathrm{OH}$ regions that wraps around the jet show finger-like structures in the wake of the jet that close toward the jet centerplane in a fashion that is reminiscent of the streamlines shown by Everett et al. (see also figure 7(b)). Furthermore, a triangular region defined by thin layers of $\mathrm{OH}$ are visible in the wake of the jet. This triangular region originates at the jet, grows further downstream and 


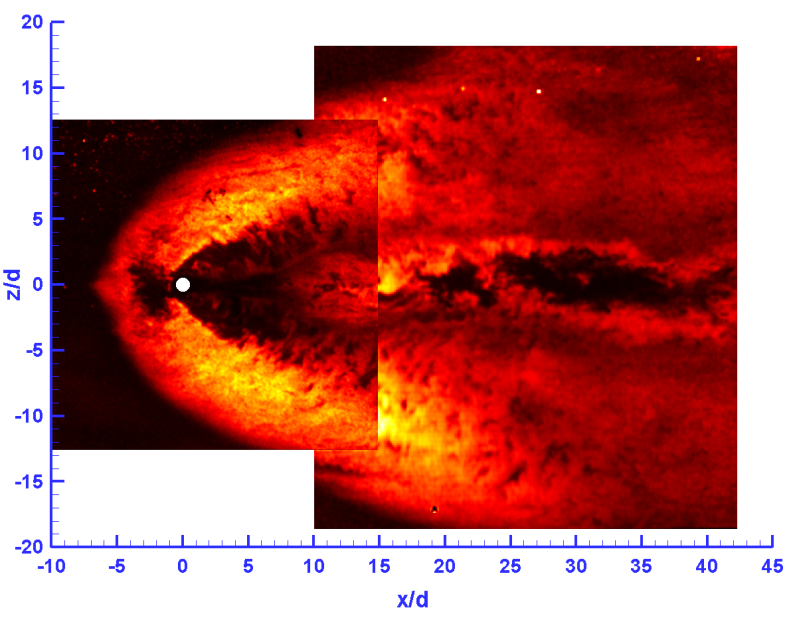

(a)

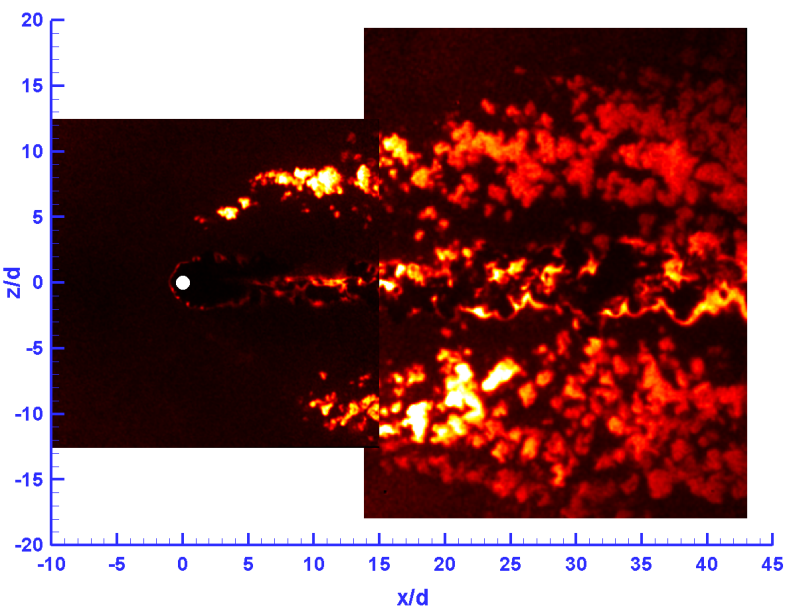

(c)

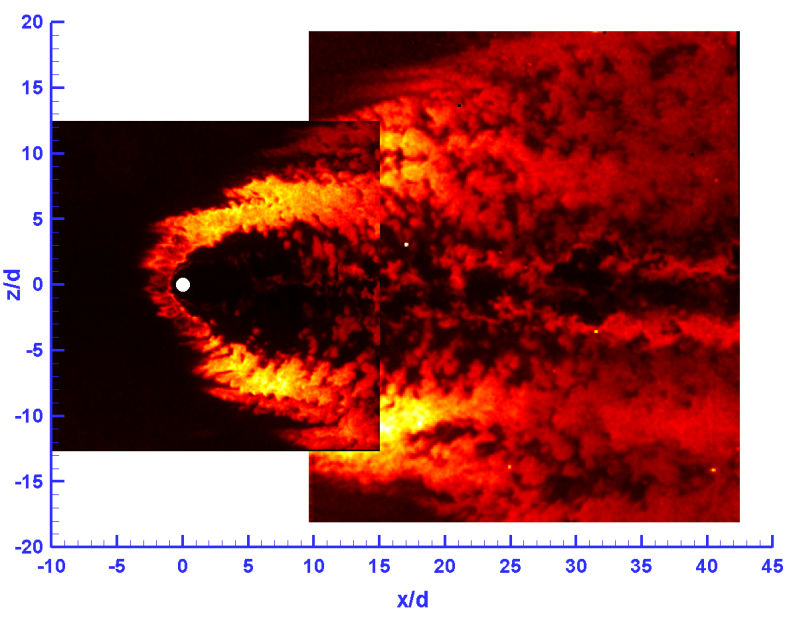

(b)

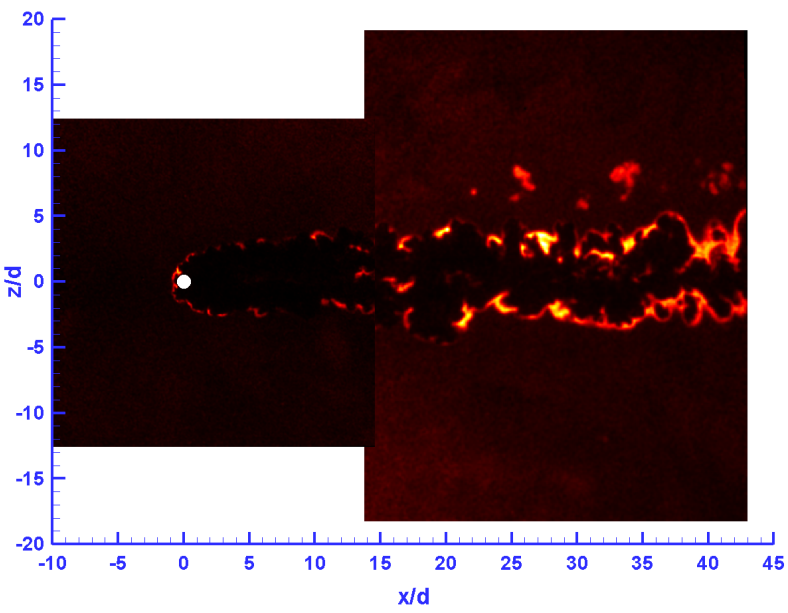

(d)

Figure 7: Instantaneous OH PLIF image on different plan-view planes for the $J=5.0 \mathrm{JISCF}$. (a) $y / d=0.25$, (b) $y / d=0.5$, (c) $y / d=1$, and (d) $y / d=2$.

then joins the wake of the jet forming what could be interpreted as a Karman vortex street. This third recirculation region in the wake of the jet defines a fuel rich region that might not experience sufficient freestream entrainment and could therefore be the origin of the unburned regions developing in the wake of the jet. The main difference with the case shown in figure 7 and the case described by Everett et al. is that the upstream recirculation region observed in the current work does wrap around the base of the jet, but does not close behind it. On the contrary, this region continuously extends to several diameters off centerline to a distance that is not consistent with the model of Everett et $a l$. and with the observations at lower values of $J$ (which seem to be more consistent with Everett et al. model). In fact, the shape of the recirculation region wrapping around the jet at low $J$ does have similarities with observations of Everett et al. but its lateral extent is not consistent with the case of $J=5.0$. It seems therefore possible that a second mechanism is responsible for the generation and spreading of the $\mathrm{OH}$ observed in the case of figure 7 . We have speculated $^{28}$ that this morphological feature is caused by a three-dimensional bow shock interaction with the boundary layer and the formation of a strongly three-dimensional process that transports fuel from the injector to the interaction region. This would also imply that this feature strongly depends on the details of the incoming boundary layer. ${ }^{28}$ 

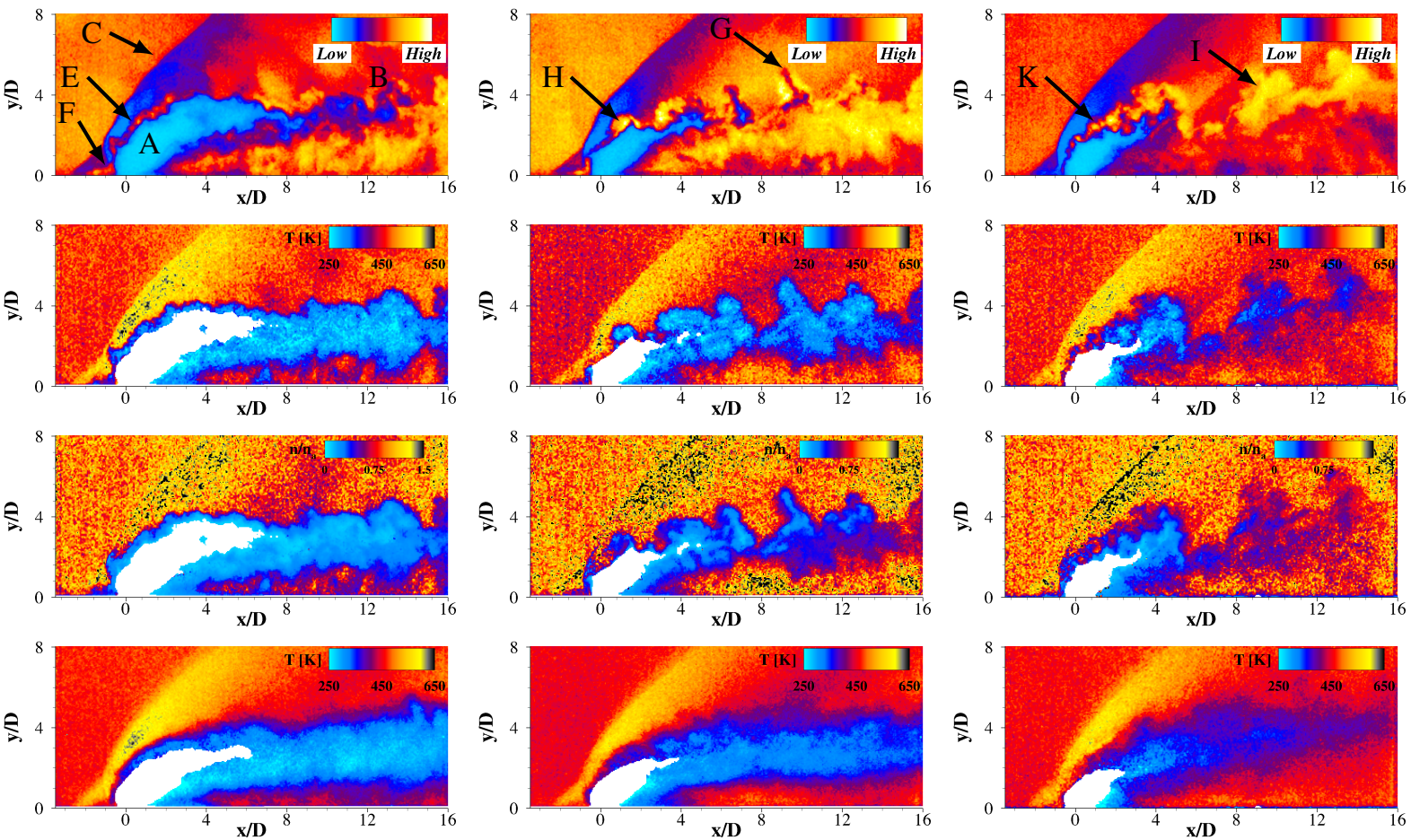

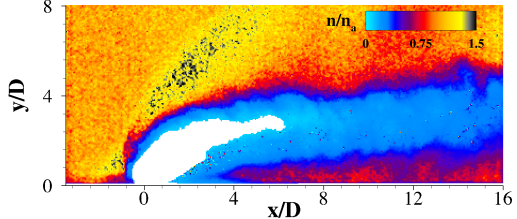

(a)

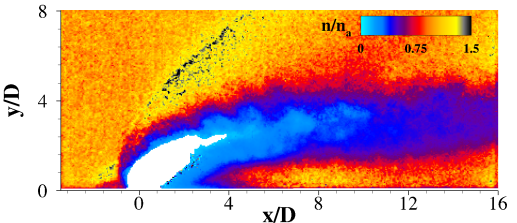

(b)

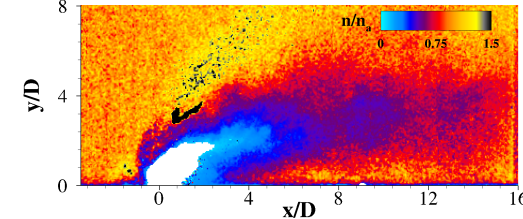

(c)

Figure 8: Instantaneous and ensemble-average turbulent structure of the transverse jet for injection of (a) $\mathrm{H}_{2}$, (b) $\mathrm{He} / \mathrm{Ar}$ mixture, and (c) Ar. First row: instantaneous, single-camera view (BP280) before conversion to temperature; second row: instantaneous temperature; third row: instantaneous (freestream-normalized) tracer number density; fourth row: ensemble-average temperature; last row: ensemble-average number density.

\section{B. Non-reacting cases: mixing properties of the JISCF using different fluids}

Figure 8 shows representative instantaneous and ensemble-averaged images of the flowfield of different JISCFs generated by injection of hydrogen (left column), Helium/Argon mixture (central column), and Argon. The top row of images refers to the PLIF images (after all necessary corrections), the second row is the reconstructed instantaneous temperature, the third row is the inferred (freestream normalized) tracer number density, the fourth column is the ensemble-average temperature, and the last column is the corresponding ensemble-average number density. All jets haves the same value of $J=2.1$. The other cases studied here show intermediate flow structures and behaviors. Analysis of multiple (single-shot) repetitions under identical conditions show similar characteristics. The ensemble-average results were computed from an ensemble of 10 single-shot experiments.

\section{Instantaneous results}

The instantaneous results on the structure of the JISCF for the three cases show substantial difference between different injection fluid types even though $J$ is held constant. A qualitative assessment suggests that the location of the nearfield, windward shear layer (feature $E$ ) is mostly unaffected by the jet fluid type, so is the location and shape (hence local strength) of the bow shock (feature $C$ in figure 8a). The upstream separation also does not appear to be affected by it and in all three cases entrainment of jet fluid into the upstream recirculation region is present $(F)$. The formation 
of this portion of the flowfield might therefore be independent of viscous and mixing effects (i.e., it is an inviscid response of the system). However, the local structure of the turbulent shear layer where mixing occurs, hence the mechanism responsible for its formation, is significantly different (compare $E, H$ and $K$ ). In turn, this also affect the far-field structure of the shear layer. In particular, the length of the core of the jet $(B)$, or more specifically the length traveled by the injected fluid without experiencing mixing, is significantly longer for hydrogen than for argon (note that the injection stagnation pressure is approximately the same for each case, which implies that the expected Mach disk height be the same ${ }^{10}$ ). For the argon case, the streamwise extent of the core is only $4 D$, while for the hydrogen case it extends up to $12-16 \mathrm{D}$. Both cases show the formation of the shear layer in the windward side. However, significant differences can be qualitatively observed in its evolution past the region of the jet where deflection occurs.

The region where (molecular level) mixing occurs is well rendered by the properties of the toluene PLIF signal (cf. PLIF images) ${ }^{57}$ In the PLIF images of figure 8, the mixing portion of the shear layer $(E, H$ and $K$ ) is rendered as the high PLIF signal portion between the core of the jet and the post-shock region (both low PLIF signal regions). In some sense, this feature corresponds to the $\mathrm{OH}$ reacting layers presented in figure 4 . For the hydrogen case, the mixing region $(E)$ is a thin and corrugated layer, which then gradually and continuously evolves into the shear layer structure of the jet far-field. For the He/Ar mixture, the shear layer evolves into large-scale vortical structures $(H)$, which then propagate downstream in the far-field and become elongated in the direction perpendicular to the local direction of the wake flow $(G)$. In fact, note the interesting finger-like structures that are clearly visible as indicated by feature $G$ of the PLIF image. For the argon case, the near-field shear layer quickly grows from small-scale structures that are readily originated at the injector exit into the large turbulent scales of the intermediate- $(K)$ and far-field $(I)$.

The LIF images alone give an idea of the level of mixing the system undergoes. However, the corresponding temperature field (second row in figure 8) demonstrates the significant differences in the mixing process and in the underlying structure of the turbulent flowfield. (The blanked out portion of the jet - also region $A-$ corresponds to regions where temperature could not be measured because of lack of tracer.) For the hydrogen case, the wake is dominated by low temperature regions, which extend all across the available measurement region. The edge of the transverse jet is well-defined, and the turbulent structures on the edge of the transverse jet can qualitatively be identified. Furthermore, low temperature regions extends from the wake of the jet down to the boundary layer and to the wall. On the contrary, the argon case shows wake temperatures near the freestream value. The low temperature regions are confined in small-scale (small compared to the hydrogen case) regions just downstream of the core of the jet, and the wake appears to break up quickly downstream. Small pockets of cold fluid are also found to exist in isolation and to penetrate into the crossflow further than what can be observed in the hydrogen case. Unlike the hydrogen case, the overall structure of the large-scale turbulent structures for the argon case seem to indicate that the dynamics of the transverse jet is dominated by vortex shedding that entrains larger amounts of crossflow fluid than the hydrogen case.

The topology of these turbulent structures that define the shear layer have a strong impact of the resulting temperature distribution (mixing) of the jet fluid, cf. the instantaneous temperature images (middle row). For the hydrogen case, the wake of the jet remains at low temperature (near the stagnation temperature of the jet) and well confined. This is a result of the low entrainment, and hence mixing, that control this case. The He/Ar mixture case, the wake is controlled by the large-scale structures identified previously and results in a corrugated outer edge. Finally, for the Ar case, past the near-field, discrete large-scale structures with what seems a periodic spacing remain well defined and control the dynamics of the wake. These features, then, result in generally higher values of the wake temperature, suggesting faster mixing.

From the results of the temperature fields shown so far, one might conclude that the argon case entrains and mixes much faster than the hydrogen case. Although below we will provide more evidence that this indeed occurs, a few clarifications are needed. Consideration of the temperature field alone to make conclusions about the underlying mixing in variable density flows using different fluids can be misleading. In fact, the resulting temperature of the mixed state is affected by both the mixture fraction (i.e., by the relative amount of cold and hot fluid), but also by the thermal properties of the mixing fluids (specifically, the specific heat $C_{p}$ ) ${ }^{57}$ Thus, to give a visualization more closely related to the mixture fraction field, the (freestream normalized) tracer number density was extracted and it is shown in figure reffig:structure (third row). The number density field provides a more direct representation of the level of mixing, but the overall structure of the transverse jets and the properties of mixing are rendered for the most part in a similar way. In any case, the final observation is that as the molecular weight of the injected fluid increases, the overall structure of the transverse jet is altered.

The observe change in the structure of the mixing layer at the windward near-field side can be attributed to an effect due to $r$. The characteristics of compressible mixing layers are found to depend on a combination of $(s, r)$ and by a compressibility parameter, which has been found to be a convective Mach number $M_{c}$ based on a suitable 


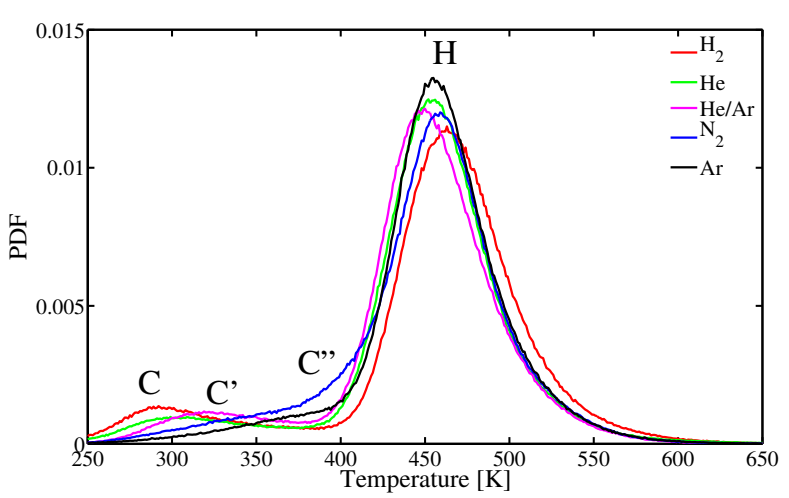

(a)

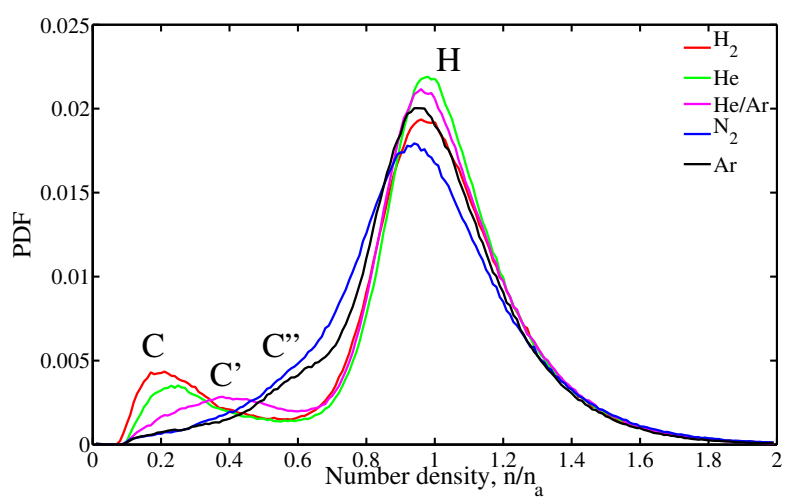

(b)

Figure 9: PDF of the (a) ensemble-average temperature for different injection fluids and (b) of the tracer number density. The PDFs are computed from all valid data point measurements in the plane.

convection velocity $U_{c}{ }^{67}$ In these experiments, going from $\mathrm{H}_{2}$ to $\mathrm{Ar}, r$ is decreased to about 0.3 (i.e., the velocity difference increases), with a corresponding increase in $M_{c}$ from about 0.1 to about 1.4 (estimated based on jet exit and freestream velocities and relative to the fastest stream between the two as defined by Papamoschou and Roshko ${ }^{67}$ ). In two-dimensional (2D) compressible mixing layers, larger velocity differences (i.e., low $r$ ) drive the mixing layer itself; however, large values of $M_{c}$ are found to decrease its growth rate, hence the underlying mixing. Applying these ideas in the Papamoschou and Roshko framework ${ }^{67}$ indicate that for the cases of these experiments the growth rate of the mixing layer should increase as we go from the $\mathrm{H}_{2}$ to $\mathrm{Ar}$ cases. This is observed in the results of figure $8-$ see $E, H, K$, although the growth rate seems to be much larger than what it would be expected by the Papamoschou and Roshko's model.

To conclude this part, a qualitative evaluation of the trajectory of the outer edge of the wake or of the largescale turbulent structures that are detected, indicates that the trajectory of these structures appear to have a weak dependance on jet fluid type, i.e., on $(r, s)$. In fact, the Ar cases appears to generated large-scale structures that penetrate marginally further into the crossflow even though the instantaneous structure is significantly different. The average overall structure of the different transverse jets can be further investigate by comparing the ensemble-average results.

\section{Ensemble-average results}

The last two rows of images in figure 8 show the ensemble-average temperature and number density distributions of the three selected cases. Note that only 10 shots were used in the averaging, and the mean results are not quite statistically converged. Nevertheless the main characteristics of the JISCF system are well rendered and for the most part both quantities provides the same information about the average structure of the transverse jets. The lack of mixing in the $\mathrm{H}_{2}$ case relative to the Ar case is readily apparent. In the former case, a long, jet-fluid rich core persists far downstream where the shear layer remains well organized; in the latter case, the core of the jet quickly breaks down (the core quickly closes in itself just downstream of the injector). Note that the masked out region refer to the core of the jet where jet-fluid rich flow exists. The Ar case shows a length of this core region substantially smaller than the $\mathrm{H}_{2}$ even though the injection pressure is nearly the same (it varies by less than 10\%). This is another indication of the faster molecular level mixing that is observed in the Ar case relative to $\mathrm{H}_{2}$ case (or other intermediate cases).

To further quantify the level of mixing provided by the different injection cases, probability density functions (PDF) of the local ensemble-average temperature and number density were computed from the ensemble-average results of figure 8. The results are shown in figure 9 and are computed from all valid points (i.e., other than points in the core of the transverse jets where measurements could not be extracted from). For all cases, we can recognize two regions: the region of high average temperature ( $H$ in figure 9 ), which corresponds to crossflow regions, and the region of low average temperature $(C)$, which corresponds to the region of the jet wake. The portion of the PDF curve that refers to the crossflow is nearly the same for all cases (other than the $\mathrm{H}_{2}$ case that shows a shifted profile) since the freestream was kept constant (and neglecting small systematic variations due to the operation of the facility, which 


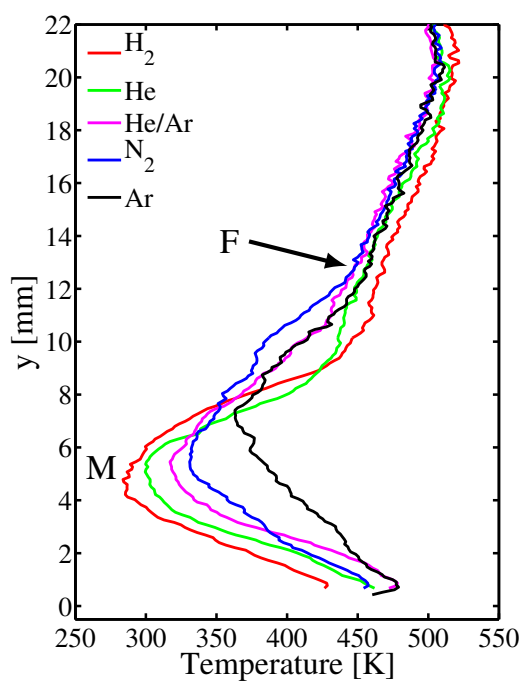

(a)

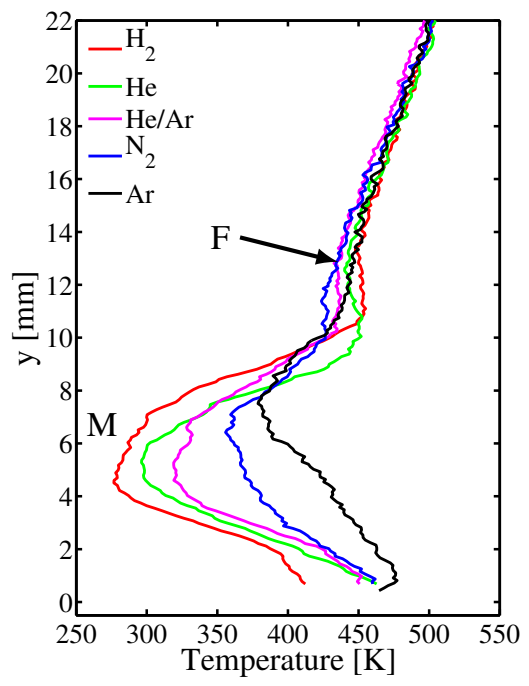

(b)

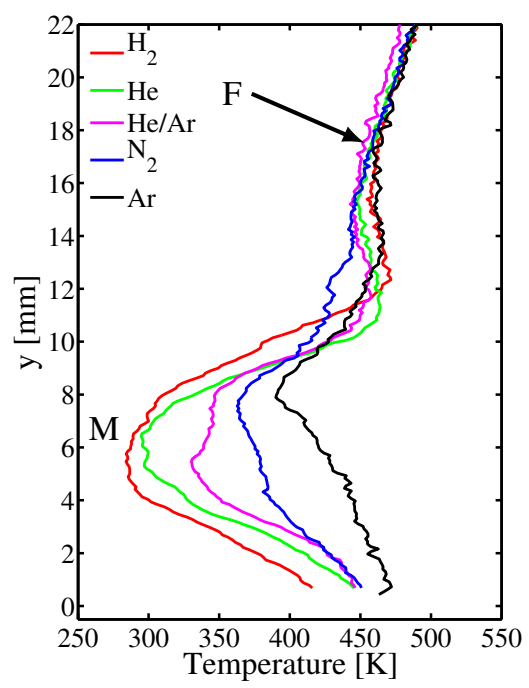

(c)

Figure 10: Vertical profile of temperature at different down locations: (a) $x / d=8.75$, (a) $x / d=11.75$, (a) $x / d=13.75$.

for example explains the shift for the $\mathrm{H}_{2}$ case).

The portion of the curve at low temperature refers to the region of the jet wake. For the $\mathrm{H}_{2}$ case, the is a second peak near $290 K$ (which is the injection temperature of the jet) - see feature $C$. For heavier jet fluids, this peaks moves at larger temperature (to $C^{\prime}$ for the He/Ar case, for example), until it is smeared out and disappears for the heavy jet cases (see $C$ " for $\mathrm{N}_{2}$ and Ar). Figure 9 further quantifies the level of mixing (in an average sense) and its dependance on the jet fluid type.

Finally, to assess the impact of injectant on the average transverse jet penetration, the average temperature and number density distributions along the $y$-direction (wall-normal) are plotted in figure 10 and 11 for the different cases and at three different downstream location $x / d$ of $8.75,11.75$ and 13.75. Because of the limited statistical convergence of the ensemble-average images, the vertical profiles presented in these figures are constructed by averaging a vertical band centered at the reported $x / d$ value and $2.5 d$ wide to suppress some of the variability. In general, both temperature and number density profiles are consistent and lead to similar conclusions. In general, the property profiles for the different fluids do not follow the same trend: the point of minimum temperature (or number density) $M$ experiences a higher temperature, the width of the profile increases and the actual minimum point move further away from the wall as higher molecular weight fluids are used. However, all profiles eventually reach a similar profile at the same wallnormal point as the freestream is approached $(F)$. Therefore, depending upon its definition, different jet penetrations can be defined. In particular, if penetration is based on point $F$, then all jets penetrates the same, but if we look at the details of the wake profile (and for example we use the point of minimum of the profile) the various fluids behave significantly different. Thus, we can conclude that the overall penetration of the jet $(F)$ is independent of the injection details but only depends on $J$; however, the exact local properties maintain a dependance on at least $r$ and $s$, at least for this limited range of fluid conditions.

\section{Discussion and conclusions}

This work looks at some of the mixing and combustion properties of transverse jets in a high-enthalpy supersonic crossflow conditions representative of scramjet engine operation. The experiments consider reacting hydrogen transverse jets and non-reacting ones. For the reacting cases, OH PLIF imaging has been used to map the instantaneous reaction zone on several different orthogonal planes. Here a summary of the more salient features are given, while more details are given elsewhere. ${ }^{27}$ In particular, the effect of jet-to-crossflow momentum flux ratio $J$ on near-wall ignition and burning, and on the ignition characteristics of the upstream separation region and near-field shear layer is investigated. The non-reacting jet cases where constructed to study the effect of velocity and density ratios (which combined define $J$ ) on the instantaneous turbulent structure, mixing properties and global characteristics to differen- 


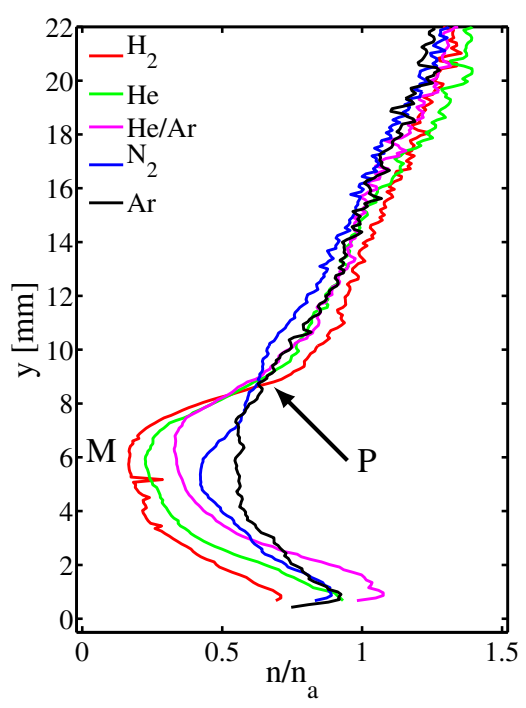

(a)

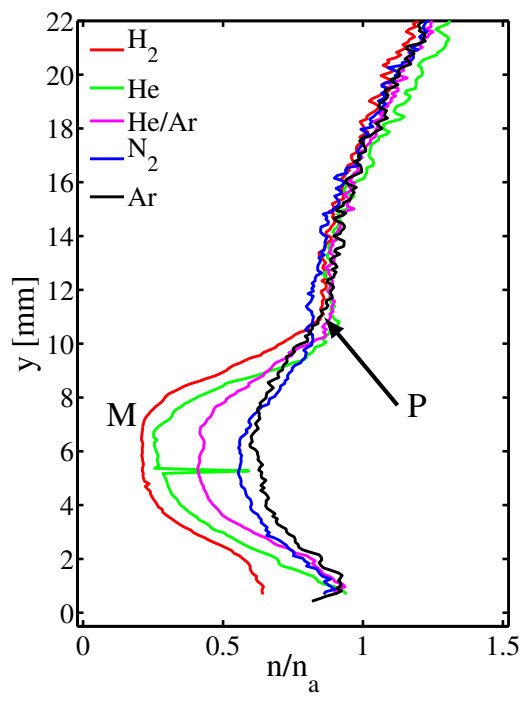

(b)

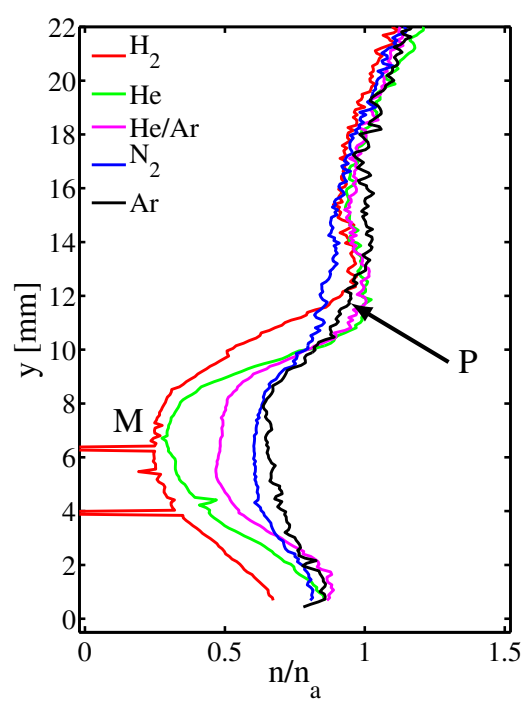

(c)

Figure 11: Vertical profile of number density at different down locations: (a) $x / d=8.75$, (a) $x / d=11.75$, (a) $x / d=$ 13.75 .

tiate effects that the parameter $J$ along cannot capture. For this purpose, different jet fluids at nominally identical $J$ and crossflow were considered. Toluene PLIF thermometry is used to infer a measure of mixing in these complex non-uniform supersonic flows. ${ }^{56,57}$

The momentum flux ratio is found to strongly affect the ignition characteristics in reacting transverse jets. Early work on characterizing and investigating transverse fuel injection for supersonic combustion ramjet applications suggested the issuing jet/bow shock interaction and the resulting separated flow as mechanisms for flame ignition, holding and stabilization. ${ }^{6}$ However, these processes are observed to occur only for sufficiently large values of $J$, at least within the range of operation considered in the study and based on previous work. ${ }^{68}$ Furthermore, it is also observed that the jet shear layer has a somewhat weak tendency of igniting and sustaining combustion. For sufficiently large values of $J$ where the transverse jet can fully emerge from the near-wall region dominated by the wake of the transverse jet, the OH PLIF imaging clearly show the large-scale rollers of the reacting layer, driven by the shear between the turning jet and free-stream flow. The thin reacting regions here appear to be similar to the reaction zone that is observed in low-speed reacting flames. ${ }^{69,70}$

Regions of the most intense $\mathrm{OH}$ are observed near the wall, across the range of $J$ values considered in the study. The point of stabilization in the wake of the jet appears to be weakly dependent on $J$ for low values and moves upstream of the injection port for large $J$. Furthermore, contrary to what analytical predictions for hydrogen/air supersonic combustion may suggest, ${ }^{71}$ the structure of the most relevant burning regions resembles more a distributed-reaction zone, maybe even (partially) premixed combustion (since the flame is effectively lifted), rather than thin sheets. These are the regions near the wall. Furthermore, it seems that, at least within the imaged region, the observed near-wall ignition and combustion might be the dominant mechanisms of flame-holding and energy deposition in the flowfield.

The reacting cases give an overall view of the combustion properties, but lack information about the underlaying mixing field. Non-reacting cases provide some of this information. The general structure of the mixing field share similarities with the corresponding

The results of the non-reacting study indicate that in spite of similar value of $J$ and similar average jet penetration, the mixing properties and the structure of the transverse jet (both instantaneous and average), have a strong dependance on injection details, i.e., on $r$ and $s$. In fact, the instantaneous turbulent structure of the jet is significantly changes from a light jet to a heavy jet case. Since these structures and their dynamics control the local entrainment and mixing processes, then mixing itself is directly affected by low (high) values of the $s(r)$ compared to the corresponding case at high (low) values. This was clearly demonstrated by the temperature and number density profiles of figures 10 and 11.

On the contrary, global properties, like overall jet penetration are less affected by $r$ and $s$, but they primary depend 
on $J$. For example, the assessment of Portz and Segal ${ }^{52}$ on JISCF penetration suggests that the average jet penetration have a very weak sensitivity to the molecular weight of the injected species and it can effectively be ignored. Their result is supported by our findings.

\section{Acknowledgments}

This paper is based on work supported by the Department of Energy sponsored Predictive Science Academic Alliance Program (PSAAP) Center at Stanford University under award number DE-FC52-08NA28614. Additionally, Victor A. Miller was supported by the Claudia and William Coleman Foundation Stanford Graduate Fellowship.

\section{References}

${ }^{1}$ Fric, T. F. and Roshko, A., "Vortical structure in the wake of a transverse jet," J. Fluid Mech., Vol. 279, 1994, pp. 1-47.

${ }^{2}$ Kelso, R. M. and Smits, A. J., "Horseshoe Vortex Systems Resulting from the Interaction between a Laminar Boundary Layer and a Transverse Jet," Physics of Fluids, Vol. 7, 1995, pp. 153-158.

${ }^{3}$ Hasselbrink, E. F. and Mungal, M. G., "Transverse Jets and Jet Flames. Part 1. Scaling Laws for Strong Transverse Jets," J. Fluid Mech., Vol. 443, 2001, pp. 1-25.

${ }^{4}$ Karagozian, A. R., "Transverse jets and their control," Progress in Energy and Combustion Science, Vol. 36, No. 5, 2010, pp. 531-553.

${ }^{5}$ Mahesh, K., "The interaction of jets with crossflow," Апnи. Rev. Fluid. Mech., Vol. 45, 2013, pp. 379-407.

${ }^{6}$ Huber, P. W., Schexnayder, C. J., and McClinton, C. R., "Criteria for self-ignition of supersonic hydrogen-air mixtures," Tech. rep., NASA TP-1457, 1979.

${ }^{7}$ Seiner, J. M., Dash, S. M., and Kenzakowski, D. C., "Historical survey on enhanced mixing in scramjet engines," J. Propul. Power, Vol. 17, No. 6, 2001, pp. 1273-1286.

${ }^{8}$ Dowdy, M. W. and Newton, J. F., "Investigation of liquid and gaseous secondary injection phenomena on a flat plate with M=2.01 to M=4.54," JPL Technical Report No. 32-542, 1963.

${ }^{9}$ Schetz, J. A., Hawkins, P. F., and Lehman, H., "Structure of highly underexpanded transverse jets in a supersonic stream," AIAA J., Vol. 5, No. 5,1967 , pp. 882-884.

${ }^{10}$ Billig, F. S., Orth, R. C., and Lasky, M., "A unified analysis of gaseous jet penetration,” AIAA J., Vol. 9, No. 6, 1971, pp. $1048-1058$.

${ }^{11}$ Cohen, L. S., Coulter, L. J., and Egan, W. J., "Penetration and mixing of multiple gas jets subjects to a crossflow," AIAA J., Vol. 9, No. 4, 1971, pp. 718-724.

${ }^{12}$ Povinelli, F. P. and Povinelli, L. A., "Correlation of Secondary Sonic and Supersonic Gaseous Jet Penetration Into Supersonic Crossflows," NASA TN D-6370, 1971.

${ }^{13}$ Papamoschou, D. and Hubbard, D. G., "Visual observations of supersonic transverse jets," Exp. Fluids, Vol. 14, 1993, pp. $468-476$.

${ }^{14}$ Gruber, M. R., Nejad, A. S., Chen, T. H., and Dutton, J. C., "Bow shock/jet interaction in compressible transverse injection flowfields," AIAA J., Vol. 34, No. 10, 1996, pp. 2191-2193.

${ }^{15}$ Everett, D. E., Woodmansee, M. A., Dutton, J. C., and Morris, M. J., "Wall pressure measurements for a sonic jet injected transversely into a supersonic crossflow," J. Propul. Power, Vol. 14, No. 6, 1998, pp. 861-868.

${ }^{16}$ Gruber, M. R., Nejad, A. S., Chen, T. H., and Dutton, J. C., "Mixing and penetration studies of sonic jets in a Mach 2 freestream," J. Propul. Power, Vol. 11, No. 2, 1995, pp. 315-323.

${ }^{17}$ Gruber, M. R. and Goss, L. P., "Surface pressure measurements in supersonic transverse injection flowfields," J. Propul. Power, Vol. 15, No. 5, 1999, pp. 633-641.

${ }^{18}$ Rogers, R. C., "A study of the mixing of hydrogen injected normal to a supersonic airstream," NASA TN D-6114, 1971.

${ }^{19}$ Santiago, J. G. and Dutton, J. C., "Velocity measurements of a jet injected into a supersonic crossflow," J. Propul. Power, Vol. 13, No. 2, 1997, pp. 264-273.

${ }^{20}$ VanLerberghe, W. M., Santiago, J. G., Dutton, J. C., and Lucht, R. P., "Mixing of a Sonic Transverse Jet Injected into a Supersonic Flow," AIAA J., Vol. 38, No. 3, 2000, pp. 470-479.

${ }^{21}$ McClinton, C. R., "Effect of ratio of wall boundary layer thickness to jet diameter on mixing of a normal hydrogen jet in a supersonic stream," NASA TM X-3030, 1974.

${ }^{22}$ Bier, K., Kappler, G., and Wilhelmi, H., "Influence of the injection conditions on the ignition of methane and hydrogen in a hot Mach 2 air stream," AIAA J., Vol. 9, No. 9, 1971, pp. 1865-1866.

${ }^{23}$ Hollo, S. D., McDaniel, J. C., and Hartfield, R. J., "Quantitative investigation of compressible mixing: staged transverse injection into Mach 2 flow," AIAA J., Vol. 32, No. 3, 1994, pp. 528-534.

${ }^{24}$ Hartfield, R. J. and Bayley, D. J., "Experimental investigation of angled injection in a compressible flow," J. Propulsion, Vol. 12, No. 2, 1995, pp. 442-445.

${ }^{25}$ Crist, S., Sherman, P. M., and Glass, D. R., "Study of the highly underexpanded sonic jet," AIAA J., Vol. 4, No. 1, 1966, pp. 68-71.

${ }^{26}$ Snyder, J. A., S., C. L., Nakakita, K., Heltsley, W. N., Mungal, M. G., and Hanson, R. K., "Pressure-sensitive paint measurements of sonic jet injection into supersonic crossflow," 43rd AIAA/ASME/SAE/ASEE Joint Propulsion Conference \& Exhibit, Cincinnati, OH, July 8-11, AIAA Paper No. AIAA-2007-5418. Paper No. AIAA-2007-5418, 2007.

${ }^{27}$ Gamba, M., Mungal, M. G., and Hanson, R. K., "Ignition and near-wall burning in transverse hydrogen jets in supersonic crossflow," 49th AIAA Aerospace Sciences Meeting and Aerospace Exposition, Jan. 4-7, Orlando, Florida. Paper No. AIAA-2011-0319, 2011.

${ }^{28}$ Gamba, M., Terrapon, V. E., Saghafian, A., Mungal, M. G., and Pitsch, H., "Assessment of the combustion characteristics of hydrogen transverse jets in supersonic crossflow," Annual Research Briefs, Center for Turbulence Research, Stanford University, 2011. 

1665.

${ }^{29}$ Schetz, J. A. and Billig, F. S., "Penetration of gaseous jets injected into a supersonic stream," J. Spacecraft, Vol. 3, No. 11, 1966, pp. 1658-

${ }^{30}$ Hersch, M., Povinelli, L. A., and Povinelli, F. P., "Optical study of sonic and supersonic jet penetration from a flat plate into a Mach 2 airstream,” Tech. rep., NASA TN D-5717, 1970.

${ }^{31}$ Torrence, M. G., "Effect of Injectant Molecular Weight on Mixing of a Normal Jet in a Mach 4 Airstream,” Tech. rep., NASA TN D-6061, 1971.

${ }^{32}$ Rogers, R. C., “Mixing of Hydrogen Injected From Multiple Injectors Normal to a Supersonic Airstream,” NASA TN D-6476, 1971.

${ }^{33} \mathrm{McDaniel}$, J. C. and Graves, J., "Laser-induced-fluorescence visualization of transverse gaseous injection in a nonreaction supersonic combustor," J. Propulsion, Vol. 4, No. 6, 1988, pp. 591-597.

${ }^{34}$ Gruber, M. R., Nejad, A. S., Chen, T. H., and Dutton, J. C., "Large structure convection velocity measurements in compressible transverse injection flowfields," Exp. Fluids, Vol. 22, 1997, pp. 397-407.

${ }^{35}$ Ben-Yakar, A., Mungal, M. G., and Hanson, R. K., "Time evolution and mixing characteristics of hydrogen and ethylene transverse jets in supersonic crossflows," Physics of Fluids, Vol. 18, 2006.

${ }^{36}$ Gruber, M. R., Nejad, A. S., Chen, T. H., and Dutton, J. C., "Compressibility effects in supersonic transverse injection flowfields," Physics of Fluids, Vol. 9, No. 5, 1997, pp. 1448-1461.

${ }^{37}$ Allen, M. G., Parker, T. E., Reineeke, W. G., Legner, H. H., Foutter, R. R., Rawlins, W. T., and Davis, S. J., "Fluorescence imaging of OH and NO in a model supersonic combustor," AIAA J., Vol. 31, No. 3, 1993, pp. 505-512.

${ }^{38}$ McMillin, B. K., Seitzman, J. M., and Hanson, R. K., "Comparison of NO and OH planar fluorescence temperature measurements in scramjet model flowfields," AIAA J., Vol. 32, No. 10, 1994, pp. 1945-1952.

${ }^{39}$ Yoshida, A. and Tsuji, H., "Supersonic combustion of hydrogen in vitiated airstream using transverse injection," AIAA J., Vol. 15, No. 4, 1977, pp. 463-464.

${ }^{40}$ Lee, M. P., McMillin, B. K., Palmer, J. L., and Hanson, R. K., "Planar fluorescence imaging of a transverse jet in a supersonic crossflow," AIAA J., Vol. 8, No. 4, 1992, pp. 729-735.

${ }^{41}$ Ben-Yakar, A. and Hanson, R. K., "Experimental investigation of flame-holding capability of hydrogen transverse jet in supersonic crossflow," Proc. Combust. Inst., Vol. 27, 1998, pp. 2173-2180.

${ }^{42}$ Rothstein, A. D. and Wantuck, P. J., "A study of normal injection of hydrogen into a heated supersonic flow using planar laser-induced fluorescence," AIAA Paper No. 1992-3423, 2004.

${ }^{43}$ Heltsley, W. N., Snyder, J. A., Cheung, C. C., Mungal, M. G., and Hanson, R. K., "Combustion Stability Regimes of Hydrogen Jets in Supersonic Crossflow," 43rd AIAA/ASME/SAE/ASEE Joint Propulsion Conference \& Exhibit, Cincinnati, OH, July 8-11, AIAA Paper No. AIAA2007-5401, 2007.

${ }^{44}$ Donbar, J. M., Gruber, M. R., Jackson, T. A., Carter, C. D., and Mathur, T., "OH planar laser-induced fluorescence imaging of hydrocarbonfueled scramjet combustor," Proc. Combust. Inst., Vol. 28, No. 1, 2000, pp. 679-687.

${ }^{45}$ Rasmussen, C. C., Dhanuka, S. K., and Driscoll, J. F., "Visualization of flameholding mechanisms in a supersonic combustor using PLIF," Proc. Combust. Inst., Vol. 31, No. 2, 2007, pp. 2505-2512.

${ }^{46}$ Ryan, M., Gruber, M. R., Carter, C. D., and Mathur, T., "Planar laser-induced fluorescence imaging of OH in a supersonic combustor fueled with ethylene and methane," Proc. Combust. Inst., Vol. 32, No. 2, 2009, pp. 2429-2436.

${ }^{47}$ Genín, F. and Menon, S., "Dynamics of sonic jet injection into supersonic crossflow," Journal of Turbulence, Vol. 11, No. 4, 2010 , pp. 1-30.

${ }^{48}$ Kawai, S. and Lele, S. K., "Large-eddy simulation of jet mixing in supersonic crossflows," AIAA J., Vol. 48, No. 9, 2010, pp. $2063-2083$.

${ }^{49}$ Boles, J. A., Edwards, J. R., and Baurle, R. A., "Hybrid LES/RANS simulation of transverse sonic injection into a Mach 2 flow," 46 th AIAA Aerospace Sciences Meeting and Exhibit, AIAA 2008-622, 2008.

${ }^{50}$ Won, S.-H., Jeung, I.-S., and Choi, J.-Y., "DES Investigation of the Ignition of Hydrogen Transverse Jet into High Enthalpy Supersonic Crossflow," 47th AIAA Aerospace Sciences Meeting, AIAA 2009-1557, 2009.

${ }^{51}$ Broadwell, J. E. and Breidenthal, R. E., "Structure and Mixing of a Transverse Jet in Incompressible Flow," J. Fluid Mech., Vol. 148, 1984, pp. 405-412.

${ }^{52}$ Portz, R. and Segal, C., "Penetration of gaseous jets in supersonic flows," AIAA J., Vol. 44, No. 10, 2006, pp. 2426-2429.

${ }^{53}$ Megerian, S., Davitian, J., De B. Alves, L. S., and Karagozian, A. R., "Transverse-jet shear layer instabilities. Part 1. Experimental studies," J. Fluid Mech., Vol. 593, 2007, pp. 93-129.

${ }^{54}$ Getsinger, D. R., Hendrickson, C., and Karagozian, A. R., "Shear layer instabilities in low-density transverse jets," Exp. Fluids, Vol. 53, 2012, pp. 783-801.

${ }^{55}$ Watanabe, J., Kouchi, T., Takita, K., and Masuya, G., "Large-eddy simulation of jet in supersonic crossflow with different injectant species," AIAA J., Vol. 50, No. 12, 2012, pp. 2765-2778.

${ }^{56}$ Miller, V. A., Gamba, M., Mungal, M. G., and Hanson, R. K., "Single- and dual-band collection toluene PLIF thermometry in supersonic flows," Exp. Fluids, , No. 54, 2013, pp. 1539.

${ }^{57}$ Gamba, M., Miller, V. A., Mungal, M. G., and Hanson, R. K., "Temperature and number density measurement in non-uniform supersonic flowfields undergoing mixing using toluene PLIF thermometry," Under review Applied Physics B, 2014.

${ }^{58}$ Heltsley, W. N., Snyder, J. A., Houle, A. J., Davidson, D., Mungal, M. G., and Hanson, R. K., "Design and Characterization of the Stanford 6 Inch Expansion Tube," 42nd AIAA/ASME/SAE/ASEE Joint Propulsion Conference \& Exhibit, 9 - 12 July, AIAA Paper No. AIAA-2006-4443, 2006.

${ }^{59}$ Örley, F., Strand, C. L., Miller, V. A., Gamba, M., and Iaccarino, G., "A study of expansion tube gas flow conditions for scramjet combustor model testing," Annual Research Briefs, Center for Turbulence Research, Stanford University, 2011.

${ }^{60}$ Örley, F., Gamba, M., Adams, N. A., and Iaccarino, G., "A study of expansion tube gas flow conditions for scramjet combustor model testing," 42nd AIAA Fluid Dynamics Conference and Exhibit, AIAA Paper No. AIAA-2012-3264, 2012.

${ }^{61}$ Gamba, M., Miller, V. A., and Mungal, M. G., "Density ratio and velocity ratio effects on the structure of transverse jets in supersonic crossflow," Int. Symposium on Turbulence and Shear Flow Phenomena, 2013.

${ }^{62}$ Trimpi, R. L., "A preliminary theoretical study of the expansion tube, a new device for producing high-enthalpy short-duration hypersonic gas flows," Tech. rep., NASA TR R-133, 1962.

${ }^{63}$ Margason, R. J., "Fifty Years of Jet in Crossflow Research,” AGARD Conference Proceeding, No. 534, 1993. 
${ }^{64} \mathrm{Su}$, L. and Mungal, M. G., "Simultaneous measurements of scalar and velocity field evolution in turbulent crosflowing jets," J. Fluid Mech., Vol. 513, 2004, pp. 1-45.

${ }^{65}$ Ben-Yakar, A., Kamel, M., Morris, C., and Hanson, R. K., "Hypersonic combustion and mixing studies using simultaneous OH PLIF and Schlieren imaging," 1998.

${ }^{66}$ Thayer, W. J., "The two-dimensional separated flow region upstream of inert and chemically reactive transverse jets," D1-82-1066, Flight Sci. Lab., Boeing Sci. Res. Lab., 1971.

${ }^{67}$ Papamoschou, D. and Roshko, A., "The compressible turbulent shear layer: an experimental study," J. Fluid Mech., Vol. 197, 1988, pp. 453477.

${ }^{68}$ Ben-Yakar, A., Experimental investigation of mixing and ignition of transverse jets in supersonic crossflows, Ph.D. thesis, Stanford University, 2000 .

${ }^{69}$ Seitzman, J. M., Ungut, A., Paul, P. H., and Hanson, R. K., "Imaging and characterization of OH structures in a nonpremixed turbulent flame," Proc. Combust. Inst., Vol. 23, 1990, pp. 637-644.

${ }^{70}$ Clemens, N. T., Paul, P. H., and Mungal, M. G., "The Structure of OH Fields in High Reynolds NumberTurbulent Jet Diffusion Flames," Comb. Sci. Tech., Vol. 129, No. 1, 1997, pp. 165-184.

${ }^{71}$ Balakrishnan, G. and Williams, F. A., "Turbulent combustion regimes for hypersonic propulsion employing hydrogen-air diffusion flames," J. Propul. Power, Vol. 10, No. 3, 1994, pp. 434-437. 